\title{
Food Applications and Physiological Effects of Anthocyanins as Functional Food Ingredients
}

\author{
Jaclyn Shipp and El-Sayed M. Abdel-Aal*
}

Guelph Food Research Centre, Agriculture and Agri-Food Canada, 93 Stone Road West, Guelph, Ontario, N1G 5C9, Canada

\begin{abstract}
Anthocyanins have been suggested as promising dietary compounds with an important role in human health. They are the largest group of water-soluble pigments in the plants, which are responsible for the red, purple and blue hues evident in fruits, vegetables, flowers and grains. As natural compounds of vegetables, fruits and red wines, anthocyanins are estimated to be widely consumed by humans with the estimated daily intake about $12.5 \mathrm{mg} / \mathrm{d}$ in the United States. Interest in anthocyanin-rich foods and extracts has intensified recently because of their possible health benefits. Anthocyanins have been shown to be potent antioxidants as well as anti-diabetic, anti-carcinogenic and having ocular effects among others. Findings of their beneficial health effects support their role as natural food colorants, functional foods and dietary supplements. The present article summarizes our knowledge on the bioavailability, antioxidant activity and healthenhancing components of anthocyanin-rich foods and extracts. In addition, their current and potential use as a natural food colorant, functional food and dietary supplement in the food and beverage industry is discussed.
\end{abstract}

Keywords: Anthocyanins, anthocyanindins, functional foods, natural health products, bioavailability, antioxidants, food colorants, dietary supplements.

\section{INTRODUCTION}

Functional foods and nutraceuticals are rapidly being integrated into the corporate mainstream and increasingly being accepted by the public due to a steady demand from consumers for healthier foods and products. With Canada being a leading producer of agricultural products, nearly 60 million tones of grains, oilseeds and specialty crops are annually produced [1]. There is great potential for the majority of these commodities to be processed into functional foods/nutraceuticals for domestic and global markets. In addition to enhancing the nutritive value and functional properties of these crops, there has been a trend towards valueadded processing and extraction of the most nutritionally valuable constituents. In this regard, specialized fractionation technologies have been developed to isolate functional and bioactive components from agricultural commodities.

Anthocyanins are of particular interest to the food colorant industry due to their ability to impart vibrant colors. Anthocyanins have been incorporated into the human diet for centuries and have been used as traditional herbal medicines due to their diverse physiological abilities to treat conditions such as hypertension, pyrexia, liver disorders, dysentery and diarrhea, urinary problems and the common cold [2]. Recently anthocyanin-containing materials are being incorporated into food products and such products require further research to demonstrate their physiological effects.

Anthocyanins (Greek anthos, flower and Greek kyanose, blue) are the largest group of water-soluble pigments in the

*Address correspondence to this author at the Guelph Food Research Centre, Agriculture and Agri-Food Canada, 93 Stone Road West, Guelph, Ontario, N1G 5C9, Canada; Tel: 519-780-8031; Fax: 519-829-2600;

E-mail: elsayed.abdelaal@agr.gc.ca plant kingdom and belong to the family of compounds known as flavonoids which are part of an even larger group of compounds known as polyphenols [3]. Anthocyanins are responsible for the red, purple and blue hues in fruits, vegetables, flowers and grains, but also play important roles in plant physiology such as attractants for insect pollinators and seed dispersal [4]. Anthocyanins are widely distributed in the human diet and the estimated daily intake has been found to be $12.5 \mathrm{mg} / \mathrm{d}$ in the United States [5]. Therefore, they can be incorporated as a functional food ingredient into our diet. Currently, anthocyanins in blue and purple corn are being used for the production of naturally colored blue tortillas [6]. Radish and potato extracts have color characteristics very similar to those of Allura red (a red dye used in food applications) [7] and therefore have the potential to be incorporated as food colorants. Incorporating anthocyanins as food colorants is not only valuable for improving overall appearance but also is very beneficial to our health.

Studies have demonstrated that anthocyanin extracts can improve sight acuteness [8-12], display antioxidative and radical-scavenging activity [13-18] and to act as chemoprotective agents [19-24]. Anthocyanins also play a role in anti-diabetic properties such as lipid lowering [25-28], insulin secretion [29-31] and vasoprotective effects [32-34]. The present paper reviews the potential health properties of anthocyanins demonstrated in studies in vitro as well as in animal and human studies. In addition, it addresses their bioavailability and potential to act as a functional food ingredient or colorant, and dietary supplement. Analysis of anthocyanins in foods and biological fluids is also discussed.

\section{CHEMICAL STRUCTURE}

Anthocyanins are glycoslyated, polyhydroxy or polymethoxy derivatives of 2-phenylbenzopyrylium containing 
two benzoyl rings (A and $\mathrm{B}$ ) separated by a heterocylic (C) ring (Fig. 1). In other words, anthocyanins are compounds of anthocyanindin (aglycone) and sugar in addition to organic acids in the case of acylated anthocyanins. Approximately 400 individual anthocyanins have been identified in plants [3]. Only six anthocyanidins are commonly found in plants including cyanidin, delphinidin, malvidin, pelargonidin, peonidin and petunidin. The glycosides of the three nonmethylated anthocyanidins (cyanidin, delphinidin and pelargonidin) are the most widespread in nature, being present in $80 \%$ of pigmented leaves, $69 \%$ of fruits and $50 \%$ of flowers [8]. They are usually bound to a saccharide residue such as glucose, galactose, rhamnose or arabinose as 3-glycosides or 3,5-diglycosides [9]. Color differences between anthocyanins are largely determined by the substitution pattern of the B-ring of the anthocyanidin, the pattern of glucosylation and the degree and nature of esterification of the sugars with aliphatic or aromatic acids and by the $\mathrm{pH}$, temperature, type of solvent and the presence of co-pigments [3]. In an aqueous solution at $\mathrm{pH} 1-3$ the flavylium cation is red colored, at $\mathrm{pH}$ 5 the resultant carbinol pseudo base is colorless and at $\mathrm{pH} 7$ 8 the blue purple quinoidal base is formed [8]. Color degradation can also occur in the presence of oxygen, light, ascorbic acid and/or metal ions and various enzymes. This is particularly important when incorporating them into foods and determining the shelf-life of the product. Since anthocyanins are highly reactive compounds which readily degrade or react with other constituents in mixtures to form colorless or brown compounds, it is necessary to increase their stability in order to retain their color and functionality. Glucosylation primarily at the $\mathrm{C}-3$ results in reduced maximum wavelength absorption but increases stability and solubility [35]. Acylation of sugar residues with cinnamic (p-coumaric, caffeic, ferulic) or aliphatic (acetic, malonic, succinic) acids further improves the anthocyanin stability [8]. Generally, 5glucosylated structures degrade more easily than 3glycosides followed by aliphatic acyl-anthocyanins and aromatic acyl derivatives [35]. Therefore, a multitude of factors can affect anthocyanins and as a result their potential as a health enhancing functional ingredient and its ability as a food colorant could be compromised.

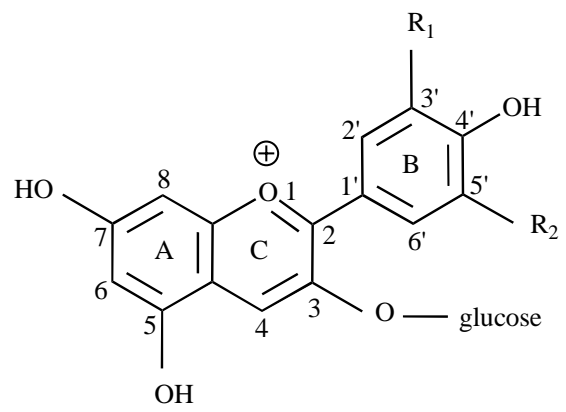

Pelargonidin-3-glucoside: $\mathrm{R}_{1}=\mathrm{H}, \mathrm{R}_{2}=\mathrm{H}$

Cyanidin-3-glucoside: $\mathrm{R}_{1}=\mathrm{OH}, \mathrm{R}_{2}=\mathrm{H}$

Delphinidin-3-glucoside: $\mathrm{R}_{1}=\mathrm{OH}, \mathrm{R}_{2}=\mathrm{OH}$

Peonidin-3-glucoside: $\mathrm{R}_{1}=\mathrm{OCH}_{3}, \mathrm{R}_{2}=\mathrm{H}$

Petunidin-3-glucoside: $\mathrm{R}_{1}=\mathrm{OCH}_{3}, \mathrm{R}_{2}=\mathrm{OH}$

Malvidin-3-rutinoside: $\mathrm{R}_{1}=\mathrm{OCH}_{3}, \mathrm{R}_{2}=\mathrm{OCH}_{3}$

Fig. (1). Structure of six common anthocyanidins in glucoside form with glucose.

\section{ANALYSIS IN FOOD AND BIOLOGICAL FLUIDS}

\subsection{Extraction of Anthocyanins}

Extraction of anthocyanins is commonly conducted with methanol or ethanol containing a small amount of acid $(15 \%$ $1.0 \mathrm{~mol} / \mathrm{L} \mathrm{HCL}$ ) with the objective of obtaining the flavylium cation form, which is stable in a highly acid medium [36]. There is no significant difference in absorbance readings or extraction efficiency between ethanol or methanol [36]. It is preferable to use ethanol since it is less toxic particularly in food use and clinical trials. Additionally, if the extract contains lipidic materials, addition of an organic solvent such as hexane to the extract can eliminate any unwanted lipid-containing substances. Acid may cause partial hydrolysis of the acyl moieties in acylated anthocyanins, especially those with dicarboxylic acids such as malonic acid, and thus the use of weak acids is advisable, such as tartaric or citric acid to keep dicarboxylic substituents intact $[37,38]$. The $\mathrm{pH}$ level has also been shown to have a significant influence on the color of the anthocyanin extracts, the absorbance readings and the extractability of the extract. At lower $\mathrm{pH}(\mathrm{pH}<2)$, blue and purple wheat extracts exhibited a red to dark red color after extraction, while at a higher $\mathrm{pH}$ $(\mathrm{pH}>4)$ extracts displayed a yellow color [36]. Additionally, absorbance readings increased with declining $\mathrm{pH}$ levels of the solvents tested (water, ethanol, and methanol). At higher $\mathrm{pH}$ levels ( $\mathrm{pH}$ 5) extractability of anthocyanin is decreased by $94 \%$ in blue and purple wheat extracts compared to extraction at a lower $\mathrm{pH}(\mathrm{pH} 1)$. Changing temperature between $25-60^{\circ} \mathrm{C}$ during extraction and storage of the extracts at $4^{\circ} \mathrm{C}$ does not seem to have a significant effect on the absorbance readings. Increasing temperature from $25^{\circ} \mathrm{C}$ to $60^{\circ} \mathrm{C}$ during extraction of purple and blue wheat increased absorbance readings by $15 \%$. Storage of blue and purple wheat anthocyanin extracts under cold conditions $\left(4^{\circ} \mathrm{C}\right)$ causes the extracts to become cloudy due to precipitation of the soluble proteins but has no significant effect on the absorbance readings [36].

Acetone has also been used for extraction of anthocyanins from red fruits $[39,40]$. Acetone allows for an efficient and more reproducible extraction and avoids problems with pectins, and permits a much lower temperature for sample concentration [40]. Garcia-Viguera et al. [39] observed that extraction of strawberries in 1\% HCL in methanol and a combination of methanol: acetic acid: water (25:1:24) all caused acetylation of anthocyanin sugars which was avoided when substituted with acetone.

\subsection{Separation and Quantification of Anthocyanins by RP-HPLC}

The most commonly employed technique today is reversed-phase high performance liquid chromatography (RPHPLC) since it permits simultaneous separation, identification and quantification of anthocyanin compounds without requiring excessive purity of the extracts [38]. Columns (internal diameter: $4.6 \mathrm{~mm}$ and length: $100-300 \mathrm{~mm}$ ) are usually maintained at ambient temperature, and elution systems are binary, using aqueous acidified solvents such as acetic acid, perchloric acid or formic acid in an organic solvent such as methanol or acetonitrile [31, 41]. Separated anthocyanins are detected and measured at $525 \mathrm{~nm}$ and the identity of anthocyanins are based on corresponding retention 
times and ultraviolet-visible (UV-vis) spectra with those of pure authentic standards such as delphinidin-3-glucoside, delphinidin-3-rutinoside, cyanidin-3-glucoside, cyanidin-3galactoside, cyanidin-3-rutinoside, peonidin-3-glucoside, petunidin-3-glucoside, pelargonidin-3-glucoside and cyanidin chloride that are commercially available. Total anthocyanin content is calculated in $u \mathrm{~g} / \mathrm{g}$ using the standard curve for either cyanidin-3-glucoside or delphinidin-3-glucoside (the most common anthocyanins in grains) [42]. The UV-vis absorbance spectrum of an anthocyanin can provide information on the nature of the anthocyanidin, glucosylation pattern and possibility of acylation [43]. Anthocyanins have a wide absorption range in the blue end of the visible spectrum with maximum absorption observed in the $500-535 \mathrm{~nm}$ regions [44]. UV-vis data has the ability to confirm some of the anthocyanidins. $U V$-vis $\lambda$ maximum for pelargonidin-based compounds has been observed around 502-506 nm, for cyanidin at $512-520 \mathrm{~nm}$, for peonidin at $517-520 \mathrm{~nm}$, for delphinidin at $525 \mathrm{~nm}$, for petunidin at 526-529 nm, and for malvidin at $530 \mathrm{~nm}$ [44].

Polarity of the anthocyanidin is the most important factor affecting the retention times. Anthocyanidins become more polar as the number of the hydroxyl group in the B-ring increases, thereby decreasing retention and become more apolar as the number of methoxyl group increases. Thus, the observed order of elution of the six most common anthocyanidins is delphindin $\rightarrow$ cyanidin $\rightarrow$ petunidin $\rightarrow$ pelargonidin $\rightarrow$ peonidin $\rightarrow$ malvidin. Sugar substitution increases the polarity with anthocyanins eluting at shorter retention times than their parent anthocyanidins with diglucosides usually eluting before the monoglycosides [43]. However, the type of sugar moiety attached influences the polarity depending on the type of sugars and their subsitution position. Acylation of the sugar moieties of the anthocyanins decreases polarity and is reflected in an increase of the retention time (malic $>$ acetic $>$ malonic $>$ succinic) $[38,43]$.

In addition, capillary electrophoresis (CE) has been used to separate ionic anthocyanin compounds by their charge [37]. The use of CE in the separation of anthocyanins is fairly new and scarce, but promising due to the high hydrosolubility of these compounds. CE is a technique which is used for anthocyanin separation and quantification. CE has been employed for the use of quantitative determination of anthocyanins in wine as a RP-HPLC alternative [45]. Various methods on CE have been developed for acylated and non-acylated anthocyanins and 3-glucoside derivatives [46], as well as for the ability to simultaneously separate 6 anthocyanins (malvidin-3-diglucoside, malvidin-3-glucoside, malvidin-3-galactoside, pelargondin-3-glucoside, cyandin3,5-diglucoside and cyanidin-3-galactoside) [47].

\subsection{Detection and Identification}

Spectral properties are often used for the characterization of anthocyanins, especially for identifying the anthocyanidin type. UV spectrometric analysis is the commonly used technique to identify and quantify anthocyanins. As described earlier anthocyanin absorption spectra depends on $\mathrm{pH}$. The maximum absorption at 520 to $540 \mathrm{~nm}$ in the visible region is the most common wavelength used in the spectrophotometric measurement of anthocyanins [41]. Mass spectrometry (MS) is a commonly used technique that permits anthocyanin identification by determining the mass of the molecular ions in the sample and of the fragments from the separation of these compounds through the application of higher ionization energies [38]. Liquid chromatographymass spectrometry (LC-MS) is used to confirm the identity of anthocyanin compounds in plants and biological fluids. LC-MS combines the separation on LC system with the selectivity and sensitivity of the MS detector allowing the identification of individual components of a mixture of compounds such as plant extracts or biological fluids [43].

Other techniques which have been used for identification of anthocyanins include electrospray ionization mass spectrometry technique (ESI-MS) and nuclear magnetic resonance (NMR) [38]. ESI-MS is used for anthocyanin characterization in complex food matrices and is especially useful for detection of low level anthocyanin metabolites in human plasma [41]. Low voltage and atmospheric pressure easily detects the positive charge of anthocyanins at low $\mathrm{pH}$ values since other potentially interfering compounds are not usually ionised. NMR is a powerful method used for structural elucidation of anthocyanins such as the acylated anthocyanins with rhamnoside derivatives found in some fruits like blackberries and minor compounds in wine analysis [37, 48]. NMR spectroscopy is a very reliable technique for the chemical analysis of food products because it is nondestructive, selective and capable of simultaneous detection of a great number of low molecular mass components in complex mixtures [48]. The use of one dimensional and two dimensional ${ }^{1} \mathrm{H}$ and ${ }^{13} \mathrm{C}$ NMR spectroscopy with large signal suppression methods allow for reliable identification for ${ }^{1} \mathrm{H}$ and ${ }^{13} \mathrm{C}$ resonance signals of individual anthocyanins in deuterated methanol $\left(\mathrm{CD}_{3} \mathrm{OD}\right)$ [48]. NMR spectroscopy is also simpler and less time consuming than LC-MS. These techniques can serve as a complement to the more widely used methods described.

\section{BIOAVAILABILITY}

Bioavailability is defined as the proportion of the nutrient that is digested, absorbed and metabolised through normal pathways [49]. It is important to food chemists to know not only how much of a nutrient is present in a food or dietary supplement, but more importantly, how much of the nutrient is bioavailable when producing a functional food or natural health product. With respect to anthocyanin bioavailability, the majority of the studies show that bioabsorption occurs quite rapidly following consumption (approx. $0.25-2 \mathrm{hr}$ ) and its excretion is complete within 6-8 hr [50-56]. Results from various in vitro, animal and human studies all consistently show that anthocyanins are absorbed in their intact glycosidic form, contrary to other flavonoids $[50,57,58]$. However, evidence shows that only less than $1 \%$ of the consumed anthocyanins are detectable in the plasma and urine [51, 53, 55].

Metabolism by the intestinal microflora is one important factor controlling the bioavailability of anthocyanins. With regards to in vitro studies, the data is limited. However in one of the few in vitro studies, Aura et al. [50] determined the bacterial metabolism of anthocyanins isolated from edible fruits and berries. Isolated cyanidin-3-glucoside and cyanidin-3-rutinoside were fermented in vitro using human faecal microbiota as inoculums and the metabolites were ana- 
lysed and characterised by RP-HPLC and LC-MS. Anthocyanins from the faecal matrix (pelargonidin-3-glucoside, delphinidin-3-glucoside and petunidin-3-glucoside) had an average recovery of $54 \pm 9 \%$ after $100 u \mathrm{~mol} / \mathrm{L}$ of the anthocyanin was incubated with $5 \%(\mathrm{w} / \mathrm{v})$ active faecal slurry for 0,6 and $24 \mathrm{hr}$. The majority of the anthocyanin glycosides disappeared within the first $\mathrm{hr}$ in the presence of $5 \%(\mathrm{w} / \mathrm{v})$ faecal slurry containing active gut microflora and only traces of different anthocyanins was detectable thereafter. In addition, formation of protocatechuic acid was detectable before the first $2 \mathrm{hr}$ as a major metabolite for both cyanidin glycosides and the detection of lower molecular weight metabolites confirmed that anthocyanins were converted by gut microflora. After $2 \mathrm{hr}$, it was evident that reconjugation of the anthocyanidin occurred with other groups. It is speculated that the low recoveries of the anthocyanins could indicate the high capacity for the faecal matrix to bind flavonoids and their metabolites. Anthocyanins could also have been converted into other metabolites which were undetectable according to Aura et al. [50] or even degraded before the bacterial conversions took place, which is possible due to the instability of the flavylium cation in alkaline $\mathrm{pH}$ values. In another in vitro study by Keppler and Humpf [54], they found that out of the six anthocyanins investigated all of the glycosides were rapidly hydrolysed by the microflora within $20 \mathrm{~min}$ and $2 \mathrm{hr}$ depending on the sugar moiety, following incubation with $100 u \mathrm{~mol} / \mathrm{L}$ of the anthocyanin for $24 \mathrm{hr}$. Similarly, because of the high instability of the anthocyanidins under physiological conditions in the intestine at a neutral $\mathrm{pH}$, primary phenolic degradation products such as phenolic acids and adlehydes were detected after only $20 \mathrm{~min}$ of incubation. Overall, it is demonstrated the rapid absorption of anthocyanin glycosides after $20 \mathrm{~min}$ to $1 \mathrm{hr}$ of incubation, followed soon after by degradation of their corresponding anthocyanidin into other compounds.

Animal experimentation with respect to bioavailability of anthocyanins has been carried out predominately in rat models focusing on anthocyanins found in fruit such as blackberries, bilberries, blackcurrant and red fruits. It has been shown that anthocyanins from red fruit extracts are incorporated in their structurally intact glycosidic forms from the digestive tract into the plasma and liver of rats after only $15 \mathrm{~min}$ of an oral supplementation of $320 \mathrm{mg}$ cyanidin-glucoside and cyanidin-3,5-diglucoside [58]. Plasma concentrations reached a peak maximum level of $1563 \mathrm{ug}$ of cyandin-glucoside and $195 u \mathrm{~g}$ of cyandin-3,5-diglucoside after supplementation. This finding was similar to other studies which also found high recoveries of anthocyanins in the plasma after 15-30 min of ingestion of anthocyanin blackcurrant juice $[55,57]$ bilberry juice [56] and red fruit anthocyanin [58]. It has also been observed that anthocyanin absorption varies greatly depending on the anthocyanin structure, with delphinidin glycosides being the highest absorbed [56]. Absorption has also been shown to differ depending on whether the anthocyanin extract consumed is purified or not. Nielson et al. [55] found that the amount of anthocyanins in the plasma in relation to dose was significantly larger $(\mathrm{p}=0.016)$ in rabbits that consumed the blackcurrant juice (anthocyanin intake $182 \mathrm{mg} / \mathrm{animal}$ ) compared to rabbits that received a dose of purified blackcurrant anthocyanins (anthocyanin intake 256 $\mathrm{mg} / \mathrm{animal}$ or $81 \mathrm{mg} / \mathrm{animal})$. This finding is validated by another study which found higher concentrations of black- berry anthocyanins $(750 u \mathrm{~mol} / \mathrm{L})$ resulted in a smaller amount of cyanidin-3-glucoside absorption compared to the smaller concentrations $(14 u \mathrm{~mol} / \mathrm{L})$ [56]. This is the first published report to suggest a presence of a food matrix effect, giving higher absorption of anthocyanins from juice than from an aqueous citric acid solution of purified anthocyanins.

Measurement of urinary excretion has also often been used to assess bioavailability of anthocyanins. With respect to the amount of anthocyanins excreted, numerous studies have shown anthocyanins to be excreted in their intact glucosylated forms. For example, in a study by Nielson et al. [55] $0.035 \%$ of anthocyanins were excreted in the urine after 30 min following ingestion of blackcurrant juice (182 mg anthocyanin/animal). Similarly, Joshi et al. [59] found that about $0.01 \%$ of grape seed proanthocaynidin extract (25-100 $\mathrm{mg} / \mathrm{kg}$ ) to be excreted in the urine. In addition, Matsumoto et al. [57] and Felgines et al. [60] found that blackcurrant anthocyanins were excreted into the urine $(0.11 \pm 0.05 \%$ of the ingested dose) as the glycoslylated form in rats after oral administration of $800 \mu \mathrm{mol} / \mathrm{kg}$ of body weight of purified anthocyanins. Talavera et al. [56] demonstrated that anthocyanins are quickly and efficiently excreted into the bile in an intact and metabolized form. Peonidin-3-glucoside was the major metabolite detected in the bile followed by cyanidin-3-glucoside. Peonidin-3-glucoside was not found in the plasma which suggests that perhaps peonidin-3-glucoside was formed in the liver and eliminated by bile rather than being incorporated into the plasma.

The majority of human experimentation studies with respect to anthocyanin bioavailability have been conducted predominantly in clinical studies using fruit extracts/mixtures. The results are consistent with the animal studies showing a rapid absorption rate in the plasma after consumption of anthocyanins and a very small amount being excreted in the urine. In a preliminary study by $\mathrm{Cao}$ and Prior [61], direct evidence was presented on the absorption of anthocyanins in their glycosidic form in humans after ingestion of $25 \mathrm{~g}$ of elderberry extract containing $1.5 \mathrm{~g}$ of anthocyanins. The peak plasma concentration after $30 \mathrm{~min}$ of consumption was $100 \mathrm{ug} / \mathrm{L}$. Matsumoto et al. [57] also found blackcurrant anthocyanin concentrate is directly absorbed in the plasma $(0.120 \mathrm{nmol} / \mathrm{L})$ and excreted in the urine $(0.11 \%$ of the ingested dose) as the glucosylated form in humans after ingestion of $33 \mathrm{mg} / \mathrm{kg}$ of body weight ( $3.58 \mathrm{mg}$ of anthocyanins) of blackcurrant anthocyanins. The extremely low bioavailability of anthocyanins has also been shown in more recent human studies. Nielsen et al. [55] found approximately $52 \mathrm{ng} / \mathrm{mL}$ and $16 \mathrm{ng} / \mathrm{mL}$ in the plasma after about $1 \mathrm{hr}$ of consumption of $4.4 \mathrm{~g}$ (1239 mg anthocyanins) and $2.7 \mathrm{~g} \mathrm{(716} \mathrm{mg}$ anthocyanins) blackcurrant juice, respectively. In another clinical study by Kay et al. [52], an average serum concentration of $591.7 \mathrm{nmol} / \mathrm{L}$ (range 197.3-986.1 $\mathrm{nmol} / \mathrm{L}$ ) was found within $2 \mathrm{hr}$ post-consumption of $20 \mathrm{~g}$ of chokeberry extracts (1.3 g cyanidin glucosides, the main anthocyanin in chokeberries). Cyanidin-3-galactoside accounted for $66 \%$ of the detected anthocyanins in the serum samples. In a latter study by Kay et al. [53], a peak plasma concentration of $96.08 \mathrm{nmol} / \mathrm{L}$ was observed after $2.8 \mathrm{hr}$ of oral ingestion of $721 \mathrm{mg}$ of cyanidin-3-glycosides from chokeberry extracts. Overall, the data consistently shows that 
the apparent bioavailability of anthocyanins is relatively low in humans with respect to plasma concentrations.

With respect to the excretion rate of anthocyanins in humans, the data shows an extremely low amount of anthocyanins excreted post-consumption. In a preliminary study, a rapid excretion rate of $5 \%$ was found after consumption of two glasses of red wine $(218 \mathrm{mg}$ of anthocyanins $/ 300 \mathrm{~mL})$ over a 12-hr period [62]. Recent findings still indicate low bioavailability of anthocyanins. Kay et al. [53] observed a total urinary excretion of anthocyanins over $24 \mathrm{hr}$ to be $1071.54 \pm 375.46 u \mathrm{~g}$, reaching a maximal rate of excretion $(202.74 u \mathrm{~g} / \mathrm{hr})$ at approximately $3.5 \mathrm{hr}$ post-consumption of $7.1 \mathrm{~g}$ of chokeberry extract (721.4 mg cyanidin-3glycosides). About $32.5 \%$ of the anthocyanins excreted in the urine were the parent compounds similar to that found in the plasma with $67.5 \%$ occurring as conjugated metabolites. The metabolites were identified as glucuronidated and methylated derivatives of cyanidin-3-glycosdies. Felgines et al. [63] reported a higher urinary excretion of $1.80 \%$ after consumption of $200 \mathrm{~g}$ of strawberries providing $179 u \mathrm{~mol} / \mathrm{L}$ pelargonidin-3-glucoside. Contrary, Felignes et al. [51] reported a urinary excretion of only $0.16 \%$ after consumption of blackberry anthocyanins (mainly cyanidin-3-glucoside). The considerable differences in urinary excretion may indicate that bioavailability is dependent on the type of individual anthocyanins ingested.

Overall, intact anthocaynin glycosides appear to be absorbed, distributed into the circulatory system and excreted in the urine. Secondly, the apparent bioavailability is consistently very low across all studies with often less than $0.1 \%$ of the ingested dose appearing in the urine. Only a small percentage of anthocyanins consumed by either animals or humans are excreted in the urine. Therefore there is a possibility that most of the anthocyanins that are consumed are absorbed in the gastrointestinal tract, specifically in the stomach and small intestine and transported into the circulatory system [54, 56]. Thirdly, absorption occurs quickly following consumption. Maximum plasma concentration is reached in 15-60 min and excretion is complete within 6-8 hr. Lastly, the amount of anthocyanin which gets absorbed seems to be dependent on the type of anthocyanin ingested. Thus, it seems difficult to confirm the apparent low bioavailability since anthocyanins exist in a number of different molecular structures and a number of potential metabolites can be generated both in vitro and in the gastrointestinal tract. Isotopically labelled anthocyanins to estimate bioavailability, transportation, accumulation into various tissues and excretion may be useful to help address this issue, but is yet to be tested. In addition, anthocyanins are likely to change form and metabolize into other alternative molecular structures such as glucuronidated and methylated derivatives of the anthocyanin and/or form corresponding phenolic acids and aldehydes. For example, Tsuda and colleagues [64] observed the concentration of protocatechuic acids to be eight times greater than that of cyanidin-3-glucoside. Therefore, future studies should focus on identifying not only the flavylium cation form but also other forms which may occur in vivo.

\section{HEALTH AND PHYSIOLOGICAL EFFECTS}

Numerous studies on anthocyanins have shown that they are not only beneficial for food applications but also as therapeutic agents. As mentioned previously, anthocyanins exhibit a wide range of therapeutic effects which have been demonstrated in various in vitro systems, animal and human trials. These therapeutic properties will be discussed in the following sections.

\subsection{Antioxidative Effects}

Dietary antioxidants can help combat reactive oxygen species and free radicals and help decrease the risk of chronic diseases such as coronary heart disease and certain cancers [13]. Anthocyanins have shown to be potent antioxidants, superior to well-known antioxidants such as butylated hydroxyanisole (BHA) [13, 65], alpha-tocopherol, 6-hydroxy-2,5,7,8-tetramethychromane-2-carboxylic acid (Trolox), catechin and quercetin [17]. There are numerous methods to evaluate antioxidant properties, however the following section will highlight the two fundamental categories of antioxidant measurement. These include radical scavenging capacity such as free radical, radical cations and radical ions, and the inhibition of lipid oxidation such as human lowdensity lipoprotein (LDL) cholesterol, methyl linoleate (MeLo), and oils. In addition, antioxidant properties of anthocyanins in vivo will be discussed as well.

\subsubsection{Radical Scavenging}

The ability of anthocyanin compounds to act as free radical scavengers against 2,2-diphenyl-1-picrylhydrazyl (DPPH) radical is one of the most common and frequently employed method. DPPH is a stable free radical due to its spare electron delocalization over the whole molecule [66]. In the presence of an antioxidant, the colored stable DPPH free radical is reduced into a nonradical DPPH-H resulting in a change of the violet color to pale yellow [13]. This discoloration is proportional to the number of electrons taken up by DPPH-H once the odd electron becomes paired off by a free radical scavenger, resulting in a decrease of absorption. This change occurs during an electron transfer reaction in which the substrate added acts as a hydrogen atom donor. The degree of radical scavenging capacity is determined by the percentage of radicals scavenged over the period of reaction time. It has been observed that the anthocyanidins/anthocyanins with the highest antioxidant activity are delphinidin, cyanidin and cyanidin-3-glucoside. These are also significantly higher than the standards alpha-tocopherol, BHA, butylated hydroxytoluene (BHT) and ascorbic acid [16]. The compounds with the lowest antioxidant activity are malvidin 3,5-diglucoside and pelargonidin 3,5-diglucoside. Monoglucosides generally have a higher scavenging capacity than the diglucosides. These results are similar to the findings obtained by Kahkonen and Heinonen [17] and Astadi et al. [14] who also found that anthocyanins possessed higher radical scavenging activity compared to the reference antioxidants (ascorbic acid, alpha-tocopherol, Trolox, BHT and rutin) in the DPPH test. Anthocyanidins, however, had slightly lower scavenging activity in the same test. According to Fukumoto et al. [16], delphinidin possessed the highest antioxidant capacity, followed by cyanidin and peonidin, pelargonidin, malvidin and petunidin. The monoglucosides were comparable to their corresponding anthocyanidin, except for peonidin-3-glucoside and pelargonidin-3-glucoside which were lower than their corresponding anthocyanidin. It was observed that cyanidin, peonidin and malvidin galacto- 
sides were weaker scavengers than their corresponding glucosides by $15-23 \%$. Cyanidin and delphindin rutinosides were also less active than their glucosides. Acylation of sugar residues with aromatic hydroxyl acids has been shown to significantly improve the radical scavenging capacity [16, 67]. In contrast to the previous two studies, Abdel-Aal et al. [13] found cyanidin-containing anthocyanins to have a higher DPPH scavenging capacity than the delphinidin-based anthocyanins. Differences in DPPH scavenging capacity between delphinidin and cyanidin-based anthcoyanins may be attributable to their molecular structure which can play a role in determining their antioxidant properties and furthermore, their bioactivity. Abdel-Aal et al. [13] and Astadi et al. [14] found the DPPH scavenging capacity of the aqueous plant extracts to be dose-dependant. These studies found that the higher the concentration of anthocyanins, the higher reducing power. For example, anthocyanin powder (anthocyanin concentrate) had a DPPH scavenging capacity 42-fold higher than the blue wheat bran [13]. Overall, all of the studies have consistently shown that anthocyanin compounds tested had a significantly high DPPH scavenging capacity comparable to the reference antioxidants. Additionally anthocyanidins lacking the $O$-diphenyl structure in the B ring (malvidin, pelargonidin, petunidin and peonidin) have a lower efficiency toward the DPPH radical compared to cyanidin and delphinidn.

The oxygen radical absorbance capacity assay (ORAC) is another reliable method to measure the antioxidant capacity of anthocyanins. It is based on the principle that antioxidant compounds present in a sample (e.g. anthocyanins) will inhibit the decay in fluorescence intensity of a fluorescent probe, fluorescein, after it is combined with 2,2'-Azobis(2methylpropionamidine) dihydrochloride (AAPH), a free radical generator that acts as an oxidizing agent [15]. Curves of fluorescence intensity versus time are recorded and the area under the curves with and without addition of an antioxidant is calculated and compared to a standard curve generated using the antioxidant Trolox, a water-soluble vitamin $\mathrm{E}$ analog. One ORAC unit is equal to the antioxidant protection given by $1 u \mathrm{~mol} / \mathrm{L}$ Trolox [15]. The ORAC values ( $u$ mol of Trolox equivalents per $100 \mathrm{~g}$, on a dry weight basis) for black and yellow (normal) barley whole grains are not significantly different from one another but are significantly higher (5430 and 5601, respectively) than the ORAC values found in the purple barley (3937) [15]. The antioxidant capacity of 14 different anthocyanin compounds has also been assessed using the ORAC assay, and compared with Trolox [68]. Among the anthocyanins, cyanidin-3-glucoside displayed the highest ORAC activity with a slope of 3.491, which was about 3.5 times stronger than Trolox. Pelargonidin had the lowest ORAC activity (slope of 1.067), but still was found to be as potent as Trolox. Overall, all anthocyanin compounds tested exhibited higher ORAC activity than Trolox, however their degree of hydroxylation and glucosylation appeared to modulate their antioxidant capacity. The anthocyanins with only one $\mathrm{OH}$ group in the $\mathrm{B}$ ring (pelargonidin, malvidin and peonidin) all had a lower ORAC activity compared to anthocyanin with 3',4'-di-OH substitution (cyanidin), with delphinidin being the exception. These findings are in agreement to that obtained by DPPH assay. Glucosylation either increased (cyanidin) or decreased (malvidin) or did not have a significant effect (pelargonidin) on the ORAC activity of the anthocyanidins.

The Trolox equivalent antioxidant capacity (TEAC) assay is based on the ability of an antioxidant to scavenge the radical cation 2,2'-azino-bis(3-ethylbenzthiazoline-6-sulfonic acid) $\left(\mathrm{ABTS}^{+*}\right)$ using spectrophotometric analysis [69]. The antioxidant reduces $\mathrm{ABTS}^{+\cdot}$ to $\mathrm{ABTS}$, decolorizing it. The TEAC value is defined as the concentration of a standard Trolox solution with the same antioxidant capacity as a $1 \mathrm{mmol} / \mathrm{L}$ concentration of a tested compound. Muselik et al. [70] determined TEAC values of anthocyanins isolated from red grape skin extract. Delphinidin-3-glucoside had the highest TEAC value $(2.93 \pm 0.05 \mathrm{mg} / \mathrm{mL})$, followed by cyanidin3-rutinoside $(2.77 \pm 0.08 \mathrm{mg} / \mathrm{mL})$, malvidin-3-glucoside $(2.45 \pm 0.03 \mathrm{mg} / \mathrm{mL})$ and petunidin-3-glucoside $(1.96 \pm 0.08$ $\mathrm{mg} / \mathrm{mL})$. The degree and position of hydroxylation and methoxylation in the $\mathrm{B}$ ring affects the stability and reactivity and thereby the antioxidant capacity of the anthocyanins as observed in the DPPH and ORAC assays. Fresh berries were macerated for 40 days prior to extraction of anthocyanins and analyzed using the TEAC assay. Fresh berry extracts exhibited TEAC value of $0.930 \pm 0.091 \mathrm{mg} / \mathrm{mL}$ which decreased to $0.915 \pm 0.050 \mathrm{mg} / \mathrm{mL}$ after 1 month of storage [69]. The TEAC value increased after 2 to 3 months of storage to $1.154 \pm 0.073 \mathrm{mg} / \mathrm{mL}$ then steadily declined to 0.952 $\pm 0.102 \mathrm{mg} / \mathrm{mL}$ and stabilized after 9 months of storage. The increase after a few months can be attributed to the hydrolysis of the flavon glycosides that results in an additional hydroxyl group that can participate in the redox reaction with the cation radical ABTS $^{+}$. The decrease of flavonoid glycosides as well as the degradation of anthocyanins after a long storage time can be responsible for the decline in the antioxidant capacity after 3 months. Sellappan et al. [71] observed high TEAC values after extraction of $10 \mathrm{~g}$ of rabbiteye blueberries, Southern highbush blueberries and blackberries. The rabbiteye blueberries had a higher average TEAC $(27.60 \pm 5.33 \mathrm{umol} / \mathrm{L} / \mathrm{g}$ fresh product $)$ compared to the Southern highbush blueberries $(14.83 \pm 8.24 \mathrm{umol} / \mathrm{L} / \mathrm{g}$ fresh product). The Georgia grown blackberries had similar TEAC on average $(20.35 \pm 3.24 \mathrm{umol} / \mathrm{L} / \mathrm{g}$ fresh product $)$ to those of the rabbiteye blueberries. Overall, the TEAC assays show that berries are a good source of antioxidants that can be used in foods and nutritional supplement formulations.

\subsubsection{Inhibition of Lipid Oxidation}

A number of lipid systems can be used to study anthocyanins ability to inhibit lipid oxidation, such as inhibition of LDL cholesterol, inhibition of MeLo or inhibition of oil or oil-containing system. LDL oxidation is one of the most studied free-radical-mediated processes occurring in the body because it is believed to play a crucial role in the formation and progression of early atherosclerotic lesions linked to cardiovascular diseases [14]. Inhibition of MeLo is not as widely studied as LDL but is still an effective method for studying antioxidant capacity of anthocyanins and other biological antioxidants [72].

\subsubsection{Inhibition of Human LDL Oxidation}

LDL does not form atherosclerotic plaques unless it is oxidised which may lead to a build up of plaque in the arteries. Therefore, the consumption of dietary antioxidants is beneficial in preventing cardiovascular diseases, particularly 
atherosclerosis [14]. Numerous studies have studied LDL oxidation in vitro, most often using cupric ion to initiate the oxidation process. For example, the antioxidant capacity of numerous anthocyanidins and anthocyanins was investigated based on the prevention of human LDL oxidation in vitro [17]. The delphinidin and cyanidin anthocyanidins were found to be the most active inhibitors followed by malvidin, peonidin, pelargonidin and petunidin. Interestingly, monoglucosides showed pro-oxidant activity (e.g. enhanced the formation of hexanal; an indicative lipid decomposition endproduct) at the lowest addition level tested $(2.5 \mathrm{umol} / \mathrm{L})$. Petunidin anthocyanin showed a slight pro-oxidant activity at all three tested concentrations $(2.5,10$ and $25 u \mathrm{~mol} / \mathrm{L})$. Overall, the glucosylated forms or anthocyanins were less active than the free forms or anthocyanidins in the prevention of LDL oxidation, except for malvidin 3, 5-diglycoside. In a more recent study by Abdel-Aal and colleagues [13], the concentration of conjugated dienes (CD) formed due to LDL oxidation in vitro dropped significantly to varying extents depending upon the type of anthocyanin. Astadi et al. [14] found similar results for all three levels of anthocyanin extracts used (20, 40 and $60 \mathrm{ppm})$. They observed a doseresponse relationship similar to the DPPH assay, with the activity being increased as the concentration of the extract increased, corresponding to a lower formation of thiobarbituric acid reactive substances (TBARS), which promote oxidative stress. The cyanidin-containing anthocyanins possessed a lower inhibition capacity against LDL oxidation compared to the delphinidin-based anthocyanins [13]. The differences in the inhibition capacity between cyanidin and delphinidin could be due to the extra hydroxyl group at the C5-position in the delphinidin structure. The additional hydroxyl group would change the release of hydrogen ions and the hydration constant $\left(\mathrm{pK}_{\mathrm{H}}\right)$, thereby increasing inhibition effects against copper-induced human LDL cholesterol oxidation [13]. This finding further emphasizes the degree to which antioxidant properties of anthocyanins can be influenced by their structure, that is, the site of glucosylation as well as type and degree of acylation with acid residues such as cinnamic and aliphatic acids. The mechanisms by which anthocyanins/anthocyanidins inhibit LDL oxidation in vitro remain unclear. It has been speculated that the protecting effects of phenolics and anthocyanins on LDL oxidation may be due to multiple factors such as scavenging of various radical species in the aqueous phase, interaction with peroxy radicals at the LDL surface and terminating chain-reactions of lipid peroxidation by scavenging lipid radicals and regenerating endogenous alpha-tocopherol back to its active antioxidative form [14, 17].

\subsubsection{Inhibition of Methyl linoleate}

The majority of antioxidant tests on flavonoids are performed in heterogeneous hydrophilic systems where oxidation is either initiated by metal ions (e.g. cupric ion) or free radicals (e.g. DPPH) [72]. The oxidation products of MeLo can be determined by RP-HPLC analysis and antioxidant capacity is expressed as the inhibition \% of the formation of MeLo-CD hydroperoxides. Kahkonen and colleagues [73] compared the antioxidant capacity of various fruits, vegetables, herbs, cereals and seeds after $72 \mathrm{hr}$ of oxidation. They found that berries having a strong hue such as bilberry, black currants, cowberries, cranberries and crowberries had a higher antioxidant capacity against the oxidation of MeLo (inhibited over 90\%) than those berries with a lighter color such as cloudberries, red raspberries and strawberries (inhibited $83-88 \%$ ). Modifications to the extraction method were shown to remarkably affect the antioxidant capacity of berries and apples [74]. The raw extracts (contain non phenolic substances such as sugars, organic acids, proteins and pigments) tested were found to be less active than the purified extracts after solid-phase extraction which was used to remove the free sugars which can interfere during antioxidant evaluation. For example, the raw acetone/water extract of raspberry inhibited the formation of MeLo hydroperoxides only by $31 \%$, whereas the purified extract gave an inhibition value as high as $93 \%$ [75]. In a latter study by Kahkonen and Heinonen [17] the activity of anthocyanidins against the oxidation of MeLo in a $10 \%$ oil-in-water system was studied. After $72 \mathrm{hr}$ a dose-dependant relationship was observed, i.e. antioxidant capacity was higher at the $250 \mathrm{umol} / \mathrm{L}$ addition level (49-88\%) compared to the $50 u \mathrm{~mol} / \mathrm{L}$ level $(38-67 \%)$. The ability to inhibit oxidation of MeLo was reported as delphinidin > malvidin > cyanidin > peonidin > pelargonidin $>$ petunidin. However, glucosylation altered the activity order, with malvidin being the strongest inhibitor followed by petunidin, cyanidin, delphinidin, pelargonidin and lastly peonidin. The type of the sugar attached also affected the activity of the monoglucosides, with no significant difference between glucoside and galactoside, but arabinoside was significantly less efficient at the $250 \mathrm{umol} / \mathrm{L}$ level.

Antioxidant behaviour is more complex when evaluated in emulsion systems than in bulk oil systems because there are more variables to influence lipid oxidation such as the type of emulsifier, $\mathrm{pH}$, and buffer system used [18]. The most common emulsifiers used in the food industry are amphiphilic proteins (e.g. casein, whey, soy or egg), phospholipids (e.g. egg or soy lecithin) and small-molecule surfactants (e.g. Spans, Tweens) [18]. For example, SanchezMoreno et al. [18] evaluated the antioxidant capacity of the phenolic compounds in 19 different commercial wines in an oil-in-water emulsion system. The oil-in-water emulsion was prepared from stripped corn oil and water and soybean lecithin as an emulsifier. The antioxidant capacity was measured by the effectiveness of the wines to inhibit the formation of $\mathrm{CD}$, and hydroperoxide. The antioxidant capacity correlated positively with the total phenol content of wines, with the antioxidant effect decreasing with increasing concentration of total phenols. For example, the inhibition of hydroperoxide formation at $10 u \mathrm{~mol} / \mathrm{L}$ gallic acid equivalents (GAE) varied from $8.4-40.2 \%$ with the red wines, while at 20 $u \mathrm{~mol} / \mathrm{L}$ GAE it only varied from $11.9-34.1 \%$. Additionally, the inhibition of hexanal formation at $10 u \mathrm{~mol} / \mathrm{L}$ and 20 $u$ mol/L GAE varied from 23.6-64.6\% and 33-46.3\%, respectively for the red wines. The antioxidant capacity of the wines was attributed to five main groups of phenolic compounds identified by RP-HPLC (benzoic acids, cinnamic acids, anthocyanins, flavan-3-ols and flavonols). The concentration of anthocyanins ranged from 9 to $158 \mathrm{mg} / \mathrm{L}$, averaging $64 \mathrm{mg} / \mathrm{L}$, being about the third most abundant compound in the wines. This finding is consistent with a similar study which evaluated the ability of three different red wine phenolic extracts $(100 \mathrm{mg} / \mathrm{L})$ to inhibit the oxidation of corn oil stripped of tocopherols [75]. The wine extracts rich in phenolic acids and flavonols exhibited high inhibitory action, 
even to a greater extent than the control BHA (200 mg/L) a well-known antioxidant. However, the wine extract rich in anthocyanins and flavanols exhibited pro-oxidant activity. The pro-oxidant activity of anthocyanins in lipids has also been observed in anthocyanidins in bulk MeLo [17]. Crude antioxidants from oat and barley malt extracts $(3300 \mathrm{ug} / \mathrm{g}$ ) have been reported to provide significantly higher antioxidant protection than the well known antioxidant BHT (approximately 1.8 and 1.2 fold higher than the 70 and $150 \mathrm{ug} / \mathrm{g}$ BHT, respectively) [76].

Overall, the in vitro studies showed that anthocyanins and their anthocyanidins, with some exceptions, are powerful antioxidants against DPPH free radical, human LDL and MeLo or oil oxidation. Their powerful antioxidant activity is comparable and sometimes even higher to well-known antioxidants such as alpha-tocopherol, Trolox, ascorbic acid, BHT, BHA and rutin. The mechanisms by which anthocyanins exert their powerful antioxidant activity remain unclear. However, as mentioned previously there are a multitude of factors which can affect their antioxidant activity. It is also proposed that radical scavenging capacity of flavonoids can be maximized if there is a free hydroxyl group in ring $\mathrm{B}$. This may explain the higher radical scavenging activity of many of the anthocyanidins compared to the glycosides in the DPPH radical scavenging test. Additionally, in lipidcontaining models such as human LDL and MeLo, glucosylation increases the polarity of the compound, thus affecting the access of the antioxidant to the lipid phase. This is supported by results by Kahkonen and Heinonen [17] who reported the diglycosides of cyanidin (3,5-diglucoside and 3rutinoside) to be less active in LDL than the corresponding 3-glucosides. However, in MeLo, glucosylation either increased (petunidin and pelargonidin), remained unchanged (malvidin and cyanidin) or decreased (delphinidin and peonidin) their activity [17].

\subsubsection{Antioxidant Activity In Vivo}

Only limited data is available on the antioxidant activity in humans. However the studies do show that following consumption of anthocyanins, serum antioxidant status is significantly increased after 4 to $24 \mathrm{hr}$ post-consumption [7779]. Youdim et al. [79] observed that blueberry polyphenolics significantly reduce red blood cells that are susceptible to reactive oxygen species (ROS) formation both in vitro and in vivo. In vitro incubation of $0.5 \mathrm{mg} / \mathrm{mL}$ of blueberry polyphenolic extract significantly enhanced red blood cell resistance to $\mathrm{H}_{2} \mathrm{O}_{2}(100 \mathrm{umol} / \mathrm{L})$ induced ROS production. This effect was also observed in vivo after 6 and $24 \mathrm{hr}$ post consumption of $100 \mathrm{mg} / \mathrm{mL}$ of blueberries. The rat's red blood cells was significantly protected against $\mathrm{H}_{2} \mathrm{O}_{2}$ induced ROS production $(\mathrm{p}<0.001)$. Mazza et al. [78] observed a direct correlation of the appearance of total anthocyanins in the serum after participants consumed $100 \mathrm{~g}$ of blueberry supplement (1.2 $\mathrm{g}$ of anthocyanins) with an increase in serum antioxidant capacity $(\mathrm{p}<0.01)$ as measured by the ORAC assay. Antioxidant capacity in human serum has also been measured using various methods following consumption of strawberries $(240 \mathrm{~g})$, spinach $(294 \mathrm{~g})$, red wine (300 $\mathrm{mL})$ or vitamin C (1250 mg) in eight elderly women [77]. Total antioxidant capacity of serum increased significantly by $7-25 \%$ during $4 \mathrm{hr}$ period after consumption of red wine, strawberries, vitamin $\mathrm{C}$ or spinach. The total antioxidant capacity of urine determined by ORAC also increased $(\mathrm{p}<0.05)$ for all the treatments except red wine, with the largest increase for vitamin C (44.9\%). The observed antioxidant protective effects against ROS and their increase in human serum indicate that the potent antioxidant activity of anthocyanins is not limited to in vitro studies, but is also observed in vivo suggesting that dietary consumption of anthocyanins is beneficial to human health.

\subsection{Ocular Effects}

The beneficial health effect that anthocyanins have on vision was one of the first reported health properties [8]. The idea that bilberry can be used to enhance night vision arose from reports of the British Royal Air Force aviators eating bilberry jam to improve their night vision during World War II [80]. The majority of the data on the effect of anthocyanins on vision were conducted under rigorous experimental conditions, including randomized, double-blind, placebocontrolled and cross-over trials. Jang et al. [9] determined the ability of bilberry extract to modulate adverse effects of $\mathrm{A} 2 \mathrm{E}$ on retinal pigment epithelial cells in vitro. $\mathrm{A} 2 \mathrm{E}$ is an auto-fluorescence pigment that accumulates in retinal pigment epithelial cells with age and can mediate a detergentlike perturbation of cell membranes and light-induced damage to the cell. This is significant since it is generally accepted that age-related macular degeneration begins with the death of retinal pigment epithelial cells, the degeneration of photoreceptor cells followed soon after by the loss of vision [9]. The results showed that the bilberry extracts were able to suppress the photooxidation of pyridinium disretinoid A2E by quenching singlet oxygen. Additionally, cells that had taken up anthocyanins also exhibited a resistance to the membrane permeabilisation that occurs because of the detergent-like action of A2E. It is important to take a step further and test the validity of these results with in vivo studies, particularly aimed at evaluating anthocyanin uptake into ocular tissues.

The role of anthocyanins on vision has also been demonstrated in a few animal studies. In a recent study by Kalt et al. [10] using a pig model, the distribution of anthocyanins in tissues such as the liver, eye and brain tissue was investigated. The results suggest that anthocyanins can accumulate in tissues, even beyond the blood-brain barrier. The highest amount of anthocyanins was found in the eye tissue with maximum concentration of $700 \mathrm{pg}$ of anthocyanins/g of fresh weight. In another animal study, Matsumoto et al. [12] investigated the ocular absorption, distribution and elimination of blackcurrant anthocyanins in rats after oral and intraperitoneal administration and in rabbits after intravenous administration. This was the first published report to demonstrate that blackcurrant anthocyanins are absorbed and distributed in ocular tissues as intact forms and pass through the blood-aqueous barriers and blood-retinal barriers in both rats and rabbits.

In a double-blind, placebo-controlled, cross-over study, Muth et al. [80] tested the ability of bilberry anthocyanins to exert a positive role on night vision using young males with good vision. After three weeks of either receiving $160 \mathrm{mg}$ of bilberry extract ( $25 \%$ anthocyanins) or placebo, the groups switched diets, and were subsquently tested on their night visual acuity and night contrast sensitivity. The data showed 
no significant difference in night visual acuity $(\mathrm{p}>0.15)$ and night contrast sensitivity $(\mathrm{p}>0.35)$ during active and placebo treatments. Therefore, the study failed to show any effect of bilberry on night visual acuity or night contrast sensitivity. Similarly, Levy and Glovinsky [81] also did not find any significant positive effects during the first $24 \mathrm{hr}$ in subjects who received either an oral dose of 12,24 or $36 \mathrm{mg}$ anthocyanins (containing $12 \mathrm{mg}$ anthocyanins as blueberry in addition to $2 \mathrm{mg}$ beta-carotene). In a recent randomized double-blind placebo-controlled study, Lee et al. [11] investigated the effect of purified high-dose anthocyanin oligomer administration on nocturnal visual function and clinical systems in low to moderate myopia subjects. There was a significant improvement in the anthocyanin group $(73.3 \%$ improved symptoms) compared to the placebo group $(\mathrm{p}<0.0001)$. In addition, the anthocyanin group showed improved contrast sensitivity levels compared to the placebo group $(\mathrm{p}<0.05)$. The differences in the results between the studies could be due to the differences in the dose of anthocyanins given. Both Muth et al. [80] and Levy and Glovinsky [81] only used a tablet which contained about $25 \%$ anthocyanins, whereas, Lee et al. [11] used a purified highdose of anthocyanin oligomer (85\%) which is significantly higher. Therefore, the observed improved effects on night vision are likely due to the increased bioavailability of the anthocyanin oligomer.

Anthocyanins from blackcurrant have also been examined for their effects on night vision and asthenopia (eye strain). In contrast to bilberry that contains 15 anthocyanins, blackcurrants only contain 4 anthocyanins: delphinidin-3glucoside; delphinidin-3-rutinoside; cyanidin-3-glucoside and cyanidin-3-rutinoside. Nakaishi et al. [82] report that an oral intake of 12.5, 20 or $50 \mathrm{mg}$ of blackcurrant anthocyanins was significant to decrease the dark adaptation threshold in a dose-dependant manner. This finding strongly suggests that blackcurrant anthocyanins are absorbed and display physiological activities. Overall, the reports on the effects of anthocyanins on vision are conflicting. The discussed in vitro, animal and human studies demonstrate that oral intake of anthocyanins or anthocyanin-rich extracts may have potential as a drug for treating opthalmological diseases such as myopia and glaucoma. However, more extensive research using animal and human intervention trials is necessary to further support and validate the data to support the described ocular health benefits.

\subsection{Anti-Obesity and Anti-Diabetic Properties}

It is suggested that a diet low in fat and high in fruits and vegetables, especially rich in polyphenols may reduce the risk of obesity and type- 2 diabetes, a condition associated with insulin resistance. Insulin resistance is a disorder in which insulin inadequately stimulates glucose transport in skeletal muscle and fat and inadequately suppresses hepatic glucose production [8]. It is still unclear which mechanisms prevent sufficient amounts of insulin to be secreted from the beta cells of the pancreas. Oral hyoglycemic agents, which directly stimulate insulin release from beta cells such as sulfonylurea-based drugs, are currently being used to overcome insulin resistance and help normalize blood glucose levels. However, like most drugs they have disadvantages such as weight gain and lack of ability to control normal blood glucose levels [8]. It is much safer and affordable to regulate blood glucose levels or elevate insulin production by consuming a diet rich in fruits and vegetables.

\subsubsection{Lipid Lowering and Oxidative Stress Properties}

Increased level of triglyceride (hypertriglyceridemia) is a major component of the insulin resistance syndrome [8]. Several studies have associated a diet high in fat with the development of type-2-diabetes and to induce hyperglycemia, hyperinsulinemia and hyperlipidemia. Recently, DeFuria et al. [25] demonstrated that blueberries can protect against whole-body insulin resistance by inhibiting the early inflammatory events in adipose tissue and can also improve glycemia in an animal model. All of the insulin-resistance related symptoms (up-regulation of inflammatory genes and increased oxidative stress) that were seen in the mice fed a high fat diet were nonexistent in the mice fed supplemented blueberry powder diet $(31.44 \mathrm{~g} / \mathrm{kg}$ dry weight of anthocyanins). This shift may be due to the ability of blueberry anthocyanins to alter mitogen-activated protein kinase and nuclear factor kappa light chain enhancer of activated B cells (NF$\left.{ }_{K} \mathrm{~B}\right)$ stress signalling pathways, which regulate cell fate and inflammatory genes. Furthermore, a decrease in hyperglycemia was found in rats fed blueberry powder coincident with reductions in adipocyte death. Similarly, Tsuda et al. [83] found that anthocyanins (cyanidin-3-glucoside) from purple corn $(2 \mathrm{~g} / \mathrm{kg}$ diet $)$ significantly suppressed the development of obesity and decreased hyperglycemia induced by a high fat diet in mice. Guo et al. [26] also demonstrated that anthocyanin-rich extract from black rice is able to eliminate the glucose intolerance and hyperlipidemia initiated by 4 weeks of a high-fructose diet fed to rats. Rats fed diets supplemented with the black rice $(5 \mathrm{~g} / \mathrm{kg}$ of diet) exhibited lower oxidative stress than the fructose-fed controls, indicated by there lower concentrations of plasma TBARS and blood oxidized glutathione. Postprandial hyperglycemic events in diabetic patients are closely associated with increased oxidative stress and is one of the most important factors in the onset and progression of vascular complications in both type- 1 and type-2 diabetes mellitus [28]. In a short-term human dietary supplementation study with red orange complex (anthocyanins, flavanones and hydroxycinnamic acids), significant improvement of thiol groups on proteins (an indirect measurement of glutathuione effect in serum) was observed [84]. Additionally, a marked decrease in serum free radical levels was demonstrated in patients with high blood oxidative stress status. Similar beneficial oxidative effects were also demonstrated in a clinical study by Rosenblat et al. [27] following consumption of $50 \mathrm{~mL}$ of pomegranate juice for 3 months. Diabetic patients who consumed pomegranate juice ( $384 \mathrm{mg} / \mathrm{L}$ anthocyanins) exhibited anti-oxidative effects such as a significant reduction in their serum lipid peroxides and TBARS by $56 \%$ and $28 \%$, respectively, and the oxidative state of their monocytes/ macrophage levels. These effects were attributed specifically to anthocyanins as demonstrated in an in vitro study by Gil and colleagues [40].

\subsubsection{Insulin Secretion}

The function of insulin is to maintain normal blood glucose levels by either suppression of glucose output from the liver or by the stimulation of glucose uptake and its metabolism [29]. Insufficient release of insulin or loss of insulin 
uptake at the tissues results in elevated glucose levels in the blood which is associated with diabetes. It is well known that dietary antioxidants including anthocyanins, protect pancreatic beta cells from glucose-induced oxidative stress [85]. Jayaprakasam et al. [29] investigated the glucose-induced insulin release from pancreatic beta-cells by anthocyanins and anthocyanidins in vitro, particularly the cyanidin, delphinidin and pelargonidn glycosides since they are the primary bioactive components of Cornus fruits (European and Asiatic Cornelian cherry). The results suggest that both anthocyanins and anthocyanidins are insulin secretagogues (enhance secretion). The most potent was delphinidin-3glucoside which significantly induced the insulin secretion at 4 and $10 \mathrm{mmol} / \mathrm{L}$ glucose concentrations as compared to the untreated cells. Cyanidin-3-glucoside, however, was more active at lower concentrations, whereas pelargonidin-3galactoside was the lowest insulin secretor. These results indicate that the number of hydroxyl groups in ring B of anthocyanins play an important role in their ability to secrete insulin. Zhang et al. [31] also reported that several compounds present in grape skin or whole grapes are capable of enhancing insulin secretion as well as selectively inhibiting cyclooxygenase- 2 enzyme. Previous studies have shown that the inducible cyclooxygenase- 2 enzyme is associated with inflammatory conditions [86]. Thus, grape skins, a waste product of the wine, may yield beneficial health effects as food additives.

A few in vitro and animal studies also investigated the role of anthocaynins in non-insulin-dependant diabetes mellitus. The retardation of alpha-glucosidase (AGH), a membrane-bound enzyme which catalyzes the cleavage of glucose from disaccharides, may be one of the most effective approaches to controlling non-insulin-dependant diabetes mellitus. An in vitro study by Matsui et al. [87], demonstrated that anthocyanin-containing plant extracts from Clitoria ternatea flowers and Ipomoea batatas purple sweet potato effectively inhibited AGH activity, suppressing the increase in postprandial glucose level. Following this study, Matsui et al. [30] demonstrated in rats, that the anthocyanin extract, caffeoylsophorose (diacylated anthocyanin) from purple sweet potato is an effective AGH inhibitor as well as a potent antioxidant. These findings indicate a substantial benefit to diabetes patients which would be obtained by developing and consumption of a functional food containing anthocyanin extracts.

\subsubsection{Vasoprotective Effects}

Different vasodilator agonists reduce endotheliumdependant vasorelaxation in several pathological conditions including diabetes [8]. One of the primary mechanisms accounting for the dysfunction of the endothelium is a decreased release of nitric oxide (NO). Extracts from red wines, other grape produce and various plants containing anthocyanins can induce endothelium-dependent vasorelaxation, via NO release or enhanced biological activity of NO. For example, red wine polyphenolic compounds have been shown to enhance the synthesis of NO resulting in induced endothelium-dependent relaxation in rat aorta [32]. Vasorelaxtion by grape products has been shown to be mediated by the NO-cGMP (guanosine 3',5'-cyclic monophosphate) pathway [88]. Blackcurrant concentrate has also been shown to induce endothelium-dependant vasorelaxation on smooth muscle in rat thoracic aorta [34]. The study demonstrated that blackcurrant concentrate $(10-20 \quad u \mathrm{~g} / \mathrm{mL}$ containing $10.83 \%$ anthocyanins) dose-dependently relaxed the norephinephrin-preconctracted aorta, but vasorelaxation was abolished by the NO scavenger oxyhemoglobin. The vasorelaxation induced by blackcurrant concentrate could be caused via the histamine $\mathrm{H}_{1}$-receptors on the endothelium, however, the identification of the exact anthocyanins having this vasorelaxation activity was not identified in these studies. These findings have been shown in other in vitro studies. Cyanidin-3-glucoside has been shown to exert a protective effect against peroxynitrite-induced endothelial dysfunction and vascular failure acting as an efficient scavenger of peroxynitrite, a pro-oxidant agent that is formed by the reaction of $\mathrm{NO}$ with superoxide anion $\left(\mathrm{O}_{2^{-}}\right)$[89]. Various other in vitro studies have also shown cyanidin's activity in the regulation of enzymes involved in NO activity. Inducible nitric oxide synthase expression was observed by Matheus et al. [33] in the palm Euterpe oleracea and by Wallerath et al. [90] from blackberry extract tested in J774 cells. Therefore, anthocyanins are able to exert vasoprotective effects as shown in the investigated in vitro studies. These effects seem attributable to the increased release of $\mathrm{NO}$ and histamine receptors particularly by cyanidin-3-glucoside. Further research is needed to validate these findings by testing the vasoprotective effects in vivo. Overall, studies suggest that anthocyanins, as a functional food component, can aid in the prevention of obesity and diabetes.

\subsection{Anticarcinogenic Effects}

Hundreds of compounds, including drugs and naturally occurring components have been identified as potential chemopreventive agents. Of those, only the compounds capable of inducing differentiation or apoptosis of cancer cells are the primary cancer chemopreventitive and/or chemotherapeutic agents [20]. Little is known regarding antioxidants and their potential as chemopreventive agents. In vitro tests have suggested that the anticancer activity may be due to anthocyanin's anti-proliferative, pro-oxidant and apoptotic effects as well as their capability to regulate gene expression [21]. Liu et al. [91] demonstrated that the proliferation of human hepatocellular liver carcinoma is inhibited in a dosedependant manner by raspberries. However, the individual compounds responsible for inhibition of the tumour cells were not identified. In a latter study by Liu et al. [24], they investigated the role of black raspberry extracts as an angiogenesis-inhibiting agent. By blocking the development of new blood vessels, it is possible to cut off the tumour's supply of oxygen and nutrients, and therefore its growth and spread to other parts of the body. The black raspberry extract $(0.075 \% \mathrm{w} / \mathrm{v})$ successfully inhibited angiogenic initiation, preventing the angiogenic switch from resting to proliferating the endothelium. It was also found to be a potent inhibitor to new vessel growth once an angiogenic intiation had taken place. However, gallic acid was the only active component identified. Further in vitro studies are needed to investigate the active anthocaynin components of this fruit. Fimognari et al. [20] identified cyanidin-3-glucoside as an anthocyanin compound capable of inducing apoptosis and cytodifferentiation in different leukemic cell lines. Additionally, treatment with cyanidin-3-glucoside was found to revert human melanoma cells from the proliferating to the differen- 
tiated state [92]. However, the effect of cyanidin-3-glucoside needs to be investigated in clinical settings to determine how the compound should be administered to maintain a steady state of the compound in the body and the dose necessary for it to act as a potential cancer chemopreventive agent.

Compared to the numerous data from in vitro studies, there are only limited published reports on animal and human studies. In an animal study, Hagiwara et al. [22] investigated the potential of purple corn to modify colorectal carcinogenesis in male rats supplemented for 36 weeks. These rats receiving the supplemented purple corn $(21.5 \%$ anthocyanins, contributing to $5 \%$ of their diet), significantly reduced the incidence $(p<0.01)$ and multiplicity of colorectal adenomas and carcinomas induced by 1,2-dimethylhydrazine (DMH) and 2-amino-1-methyl-6-phenylimidazo (4,5-beta) pyridine (PhIP). In a comparative investigation by Kang et al. [23], the mice received either a mixture of anthocyanins at $800 \mathrm{mg} / \mathrm{L}$ or pure cyanidin at $200 \mathrm{mg} / \mathrm{L}$ with drinking water or tart cherries added to the diet $(200 \mathrm{~g} / \mathrm{kg}$ diet $)$. The amount of anthocyanins and cherries that the mice consumed equals approximately 2.4 and $0.6 \mathrm{mg}$ anthocyanins/animal/day or $600 \mathrm{mg}$ of tart cherries/animal/day, respectively. The animals which received the supplemented diet had significantly reduced caecal adenomas compared to the animals on the control diet or those that received the nonsteroidal anti-inflammatory drug sulindac [23]. Studies have also investigated the cancer preventative properties of chokeberry, bilberry, grapes and black raspberries [93]. Animals which received anthocyanin-rich extracts of chokeberry, bilberry or grape at $4 \mathrm{~g} / \mathrm{kg}$ diet (approx $35 \mathrm{mg} / \mathrm{animal} /$ day) for one week before the carcinogen (azoxymethaneinduced colonic aberrant crypt foci) had significantly reduced number of aberrant crypt foci compared to the control rats. The reduction was also accompanied with inhibition of cyclooxygenase-2-gene expression in malignant tissue. Similarly, rats who consumed approximately $0.38,0.75$ or $1.5 \mathrm{~g}$ black raspberries/animal/day decreased the multiplicity of azoxymethane-induced aberrant crypt foci by $21-36 \%$ and adenocarcinomas by $28-80 \%$ [94]. Anthocyanins have also been shown to prevent skin cancer in rodents. Topical application of anthocyanin-containing pomegranate extract elicited a delay in onset and decrease in incidence and burden of skin tumours [95].

The epidemiological data is not abundant, but there are reports suggesting cancer preventative properties of anthocyanin-containing fruits and vegetables. In an earlier elderly cohort study, those who consumed strawberries less than once/week had an odds ratio of 0.3 for developing cancer at any site compared to subjects who did not consume high berry consumption [19]. Other epidemiological studies have linked colored fruits and vegetables to a lower incidence of human breast cancer [96] and colorectal polyp recurrence [97]. Despite the data suggesting that anthocyanin containing fruits and vegetables can help reduce and delay the onset of different types of cancers (liver, leukemia, colorectal, skin and breast cancer), further studies are necessary to demonstrate the effectiveness of their clinical development in order to be used in chemopreventive interventions. Studies should focus on both the specific anthocyanidin(s) and/or anthocyanin(s) worthy of development as well as on the tissue types that might be particularly susceptible to cancer prevention by anthocyanins.

\subsection{Other Physiological Effects}

Anthocyanins have also been shown to prevent the risk of cardiovascular disease and coronary heart disease by moderate consumption of red wine [98-100]. Approximately 2 glasses of red wine per day ( $>22 \mathrm{~g} /$ day) has been associated with a $27-39 \%$ reduced risk for both cardiovascular disease and coronary heart disease [100]. Anthocyanins have also been shown to have protective effects on gastric damage, which is closely related to their ability to prevent or ameliorate the inflammatory process [101]. Black chokeberry red pigment supplementation of $30-300 \mathrm{mg} / \mathrm{kg}$ of body weight to rats have shown a significant protective effect on gastric mucosa in a dose-dependant manner. Administration of 30 $\mathrm{mg} / \mathrm{kg}$ of body weight could effectively suppress ethanolinduced gastric mucosal damage by $\sim 50 \%$ [101]. They also found to play a role in the treatment of various blood circulation disorders resulting from capillary fragility [68], inhibition of platelet aggregation [102] and radiation-protective agents [103].

\section{FOOD APPLICATIONS}

Consumers predetermine their expectation of flavor and taste by the color of the food product. The food industry has used colorants for centuries to enhance or restore original appearance of foods or to ensure uniformity as indicator of food quality [7]. Therefore, color is a major issue for the food industry and the manufacturer will try its best to retain the natural appearance of the raw material. However, during processing and storage, food color may be altered through the action of light, temperature, oxygen, metal ions and endogenous enzymes as previously described in the structure of anthocyanin. In addition, the color of fruits and vegetables will vary during seasons depending on their intra- and interspecific variables and soil conditions at the site of cultivation and post-harvest treatments [35]. Currently, more consumers are health-conscience and becoming increasingly more concerned about what they are eating and as a result, synthetic pigments are becoming increasingly rejected by the consumer. The acceptance of natural or nature-derived alternatives such as anthocyanin components is promoted due to their increasing health benefits. Extensive research on the health benefits of anthocyanins has drawn attention to their use in functional foods and as a natural food colorant. For example, purple corn has been used as a food colorant since 1977 [6] and red rice is used as a functional food in China and is commonly used as a food colorant in bread, ice cream and liquor [104].

\subsection{Anthocyanins as Food Colorants}

A good hygienic status and high color stability and purity along with a faint flavor and taste are the attractive characteristics of a promising food color source. Additional criteria such as good availability, a low price and high yield crops whose pigments possess an acceptable tinctorial strength are also valued [35]. A major drawback of anthocyanins as food colorants is their decrease in tinctorial strength in low acid media. However, research in the development of anthocyanin containing food colorants has identified glucosylation and acylation to greatly improve the stability of the anthocyanin to $\mathrm{pH}$ changes, heat treatment and light exposure [35]. 
Vegetable sources such as radish, purple sweet potato, black carrot, red-fleshed potato or red cabbage have been shown to provide a higher percentage of acylated anthocyanins than fruits which reflect a higher tinctorial strength of the respective extracts at food $\mathrm{pH}$ [35]. Of these, radishes and red potatoes in particular have the potential to be used as an alternative for Federal Food Drug and Cosmetic Red No. 40 (Allura red). For example, color measurements showed that acylated pelargonidin derivatives extracted from red radishes imparted a red color to maraschino cherries close to that of Allura red at $\mathrm{pH}$ 3.5. Conversely, when anthocyanins are 5glucosylated such as in red cabbage, they tend to lose their color more easily, which is characterized by their hydration constants, with higher values corresponding to higher coloring efficiencies at a given $\mathrm{pH}$ [105]. The color dilution assay developed by Hofmann [107] is an efficient way of ranking the tinctorial power of isolated pigments in wine, fruit and vegetable extracts. Horticulturalists should focus on breeding for pigment quality of crops using this color efficiency test in addition to breeding for maximum yields. For example, black carrot has been shown to be a good anthocaynin source not only for its good taste and color, but also because of its high ratio of monoacylated structures increasing color retention at food $\mathrm{pH}$ as well as small amounts of non-anthocyanic phenolics which are known to oxidize easily [105]. Red wine has been shown to be an exceptional source for food coloring. Its co-pigments are reduced to form stable complexes during fermentation and generated intermediate products further react to yield color stable anthocyanin derivatives [106]. Red rice is commonly used as a food colorant in China [104]. Red rice is approved by the Chinese Ministry of Health as a modern food additive to increase the color and delicacy of meat, fish and soybean products as part of the Chinese diet [108]. Successful food applications have also been reported for red cabbage and radish extracts, made only possible after the development of suitable procedures to remove or drastically reduce the concentration of aroma and flavour compounds [7]. Other applications of anthocyanin extracts include coloration of acid fruit preparations, jams and preserves. However, the use of extracts in these applications is highly dependable on the nature and quality of the fruit (fresh, frozen or sulphited) and whether proteins are present. For example, extracts containing other phenolics or oliogomeric pigments above a certain level cannot be used to color jellies as they form precipitates with gelatine [109]. In confectionary sugar, grape extract $(0.4 \% \mathrm{w} / \mathrm{w})$ produces a clear ruby red color in boiled sweets, which can be adjusted with colorants from other fruit sources. Giusti and Wrolstad [7] investigated the viability of acylated anthocyanins from red radish, red cabbage, black carrot and grape skin extract to color dairy products such as yogurt and sour cream having $\mathrm{pH}$ levels around 4.2-4.5. They discovered that radish and carrot alone or in combination could provide a desirable red hue for dairy applications at concentrations as low as $5 \mathrm{mg}$ monomeric anthocyanin/100 g sample. Since the shelf-life of these tested dairy products are only a few weeks under refrigeration, the stability of anthocyanin extracts would not be affected making them viable alternatives. With increasing $\mathrm{pH}$ value, discoloration occurs, but if the product being colored contains components capable of acting as co-pigments, color may be retained and also light-stabilised to a certain extent [109]. Therefore, the use of acylated anthocyanins with improved color and stability to heat, light and $\mathrm{pH}$ could hold a promise for incorporating anthocyanins as natural food colors. It is predicated that in the future, the production and adding of anthocyanins as natural food colorants will steadily increase, following the current trend away from synthetic colors [41].

Regulatory policies concerning the use of anthocyaninbased colorants differ considerably from country to country. Many countries limit the approval of colorant usage to defined food products, and do not permit their use in such commodities as milk and fruit juice. The European Union, Australia, Canada, Cyprus, Finland, Japan, New Zealand, Norway, South Africa, Sweden, Switzerland, and the USA restrict anthocyanin usage to defined food products [110]. Countries that do have general approval for food use of anthocyanin colorants are Chile, Columbia, Iran, Israel, South Korea, Malta, Peru, Saudi Arabia and the United Arab Emirates. In the European Union, all anthocyanin-derived colorants are recognized as natural colorants under classification E 13. In the U.S., 4 of the 26 colorants that are exempt from certification and approved for food use are anthocyaninderived: grape skin extract, grape color extract, fruit juice, and vegetable juice [110]. In addition to grapes, the more common fruit and vegetable sources include red cabbage, blackcurrants, radishes, elderberries, chokeberries, blackberries, black raspberries, and black currants. Blue wheat could also be a promising source for the production of anthocyanin-based colorants [13].

\subsection{Anthocyanins as Functional Food Ingredients}

Anthocyanins are accumulated mostly in flowers and fruits, but are also present in leaves, stems, storage organs and grains [41]. Various berries and black currants are the richest sources of anthocyanins, although eggplant and purple and blue pigmented grains also contain high amounts of anthocyanins. The widespread occurrence of anthocyanins in fruits, vegetables and red wines results in a high ingestion by humans. Depending on the country and nutritional habits of the individuals, the daily intake of anthocyanins has been estimated to range from several milligrams to hundreds of milligrams per person [41]. Intake of anthocyanins is steadily increasing because extracts and juices from fruits and vegetables with high anthocyanin contents are becoming much more commercially available nowadays, and health benefits of anthocyanins have become well evident.

Anthocyanin applications in food systems are preferably used in acidic food to assure a predominance of the flavylium cation. For example, blue wheat anthocyanins, either in whole meal or isolated form are most thermally stable at $\mathrm{pH} 1$, and their degradation is insignificantly lower at $\mathrm{pH} 2$ as compared to $\mathrm{pH} 5$ [42]. This could explain the main use of anthocyanin extracts such as grape pigments in beverages and soft drinks approximately $3 \mathrm{~kg}$ of a $1 \%$ anthocyanin extract added to $1000 \mathrm{~L}$ of beverage can impart a deep red color [109]. Grape extracts have also been shown to be resistant to sulphur dioxide $\left(\mathrm{SO}_{2}\right)$ bleaching [109]. Addition of $\mathrm{SO}_{2}$ is widely used as a preservative in beverages which may cause anthocyanin color to be bleached initially, but then partially restored as $\mathrm{SO}_{2}$ becomes oxidised [109]. It has also been demonstrated that the addition of $\mathrm{SO}_{2}$ during heating of blue wheat increased stabilization of the antho- 
cyanin pigments compared to the control sample or heating without $\mathrm{SO}_{2}$ [42].

Currently, most of the colored corn is used for ornamentation due to its colorful appearance with only a small amount being utilized in the production of blue and pink colored tortillas. Purple wheat is crushed into large pieces, which are spread over the exterior of multigrain bread [111]. Additionally, Abdel-Aal et al. [44] reported that colored corn grains such as blue, pink and purple have relatively high amounts of anthocyanins, especially purple corn $(1277 u \mathrm{~g} / \mathrm{g})$ holding a promise for the development of functional foods and/or natural colorants. Similar to other bioactive compounds, the environment in which they are grown was determined to have a significant influence on their anthocyanin concentration and composition. Therefore, during crop production efforts should be made to ensure maximum anthocyanin content for both fruits and cereals. Special sorghum such as black sorghum has also been shown to contain significant levels of anthocyanins and other phenols concentrated in their brans [112]. Black sorghum is reported to have significantly more anthocyanin pigments than other sorghums. Black sorghum bran fraction was found to contain approximately $4.0-9.8 \mathrm{mg} / \mathrm{g}$ of anthocyanins mainly 3deoxyanthocyanidins such as luteolinidin and apigeninidin which is relatively high compared to pigmented fruits and vegetables $(0.2-10 \mathrm{mg} / \mathrm{g})$ on a fresh weight basis [112]. Therefore, black sorghum has a good potential as a functional food product. Red rice is also used as a functional food in China to treat abdominal pain and aid in control of cardiovascular disease [104].

\subsection{Anthocyanins as Dietary Supplements}

Anthocyanins-rich food extracts have been incorporated and developed into a dietary food supplement. For example, anthocyanin extracts from purple corn have been incorporated as an antioxidant dietary supplement with claims to promote healthier, younger looking, more radiant beautiful skin [113]. Anthocyanins are also being sold as supplement called Medox which incorporates a concentrated amount of cyanidin-3 glucoside and delphinidin-3-glucoside extracted from Norweigan bilberries (Vaccinium myrtillus) and black currants (Ribes nigrum) [114]. Red rice is also being fermented and sold as a dietary supplement and marketed as Cholestin to help reduce cholesterol levels [115]. Additionally, blue wheat bran can be furthered processed to produce anthocyanin-rich blue wheat powder as a dietary supplement [13].

Overall, anthocyanins are gradually being incorporated into the food and beverage products as a food colorant, as a functional food or a dietary supplement. Increased development of anthocyanins with enhanced stability and prolonged shelf life will increase food applications and overall consumption and thereby increase its positive role in human health.

\section{CONCLUSIONS}

In summary, in vitro and in vivo studies have provided significant evidence of the excellent potential for the development of anthocyanins as a functional food and/or dietary supplement. Rapid advancements in food technology and analysis have allowed for efficient extraction, processing and identification of anthocyanin compounds from various fruits, vegetables and grains to be increasingly incorporated into the food and beverage industry as food colorants, functional foods and dietary supplements. In addition, it is important to understand the nature of absorption and metabolism of anthocyanins in vivo. Improved understanding of these processes will enable the development of new food products, both fresh and manufactured, with greater therapeutic efficacy. Currently, relatively little is known about absorption of anthocyanin compounds after consumption and of the mechanisms by which they exert their beneficial health effects. The available data has shown that anthocyanins are absorbed and excreted in the urine. More in vivo studies are needed to explore their absorption and distribution, as well as effects of intestinal microflora on their structure and gut flora metabolites, and effects of long term exposure of anthocyanins. Increased knowledge of their bioavailability and therapeutic effects will result in better adoption of anthocyanin-based products as functional foods. Currently, only a small percentage of anthocyanin-containing fruits, vegetables and grains are being integrated into the food and beverage industry. Thus, greater publicity of the health benefits of anthocyanins should increase consumption of these products and thus, their development and use as a functional food, food colorant and dietary supplement.
ABBREVIATIONS
AAPH $=2,2$-Azobis(2-methylpropionamidine) dihy- drochloride
ABTS $^{+}=2,2^{\prime}$-azino-bis(3-ethylbenzthiazoline-6- sulfonic acid)
$\mathrm{AGH}=$ Alpha-glucosidase
BHA $=$ Butylated hydroxyanisole
BHT = Butylated hydroxytoluene
$\mathrm{CD}=$ Conjugated dienes
$\mathrm{CE}=$ Capillary electrophoresis
$\mathrm{DPPH} \quad=$ 2,2-diphenyl-1-picrylhydrazyl
ESI-MS = Electrospray ionization mass spectrometry technique
GAE $=$ Gallic acid equivalents
LC-MS = Liquid chromatography-mass spectrometry
LDL = Low-density lipoprotein
MeLo = Methyl linoleate
MS $=$ Mass spectrometry
NMR $=$ Nuclear magnetic resonance
ORAC $=$ Oxygen radical absorbance capacity assay
ROS $=$ Reactive oxygen species
RP-HPLC $=$ Reversed phase high performance liquid chromatography
TBARS $=$ THIOBARBITURIC acid reactive substances
TEAC $=$ Trolox equivalent antioxidant capacity
Trolox = 6-hydroxy-2,5,7,8-tetramethychromane-2- carboxylic acid
UV-vis = Ultraviolet-visible 


\section{REFERENCES}

[1] Oomah BD, Mazza G. Health benefits of phytochemicals from selected Canadian crops. Trends Food Sci Tech 1999; 10: 193-198.

[2] Konczak I, Zhang W. Anthocyanins - more than nature's colours. J Biomed Biotechnol 2004; 5: 239-240.

[3] Mazza G. Anthocyanins and heart health. Ann Ist Super Sanita 2007; 43: 369-374.

[4] Schemske DW, Bradshaw HD. Pollinator preference and the evolution of floral traits in monkeyflowers (Mimulus). Proc Natl Acad Sci USA 1999; 96: 11910-11915.

[5] Wu X, Beecher GR, Holden JM, Haytowitz DB, Gebhardt SE, Prior RL. Concentrations of anthocyanins in common foods in the United States and estimation of normal consumption. J Agric Food Chem 2006; 54: 4069-4075.

[6] Sugiyama Chemical Institute. Anthocyanin food colouring agent from purple corn. Japanese Patent 77130824. 1977.

[7] Giusti MM, Wrolstad RE. Acylated anthocyanins from edible sources and their applications in food systems. Biochem Eng $\mathbf{J}$ 2003; 14: 217-225.

[8] Ghosh D, Konishi T. Anthocyanins and anthocyanin-rich extracts: role in diabetes and eye function. Asia Pac J Clin Nutr 2007; 16: 200-208.

[9] Jang YP, Zhou J, Nakanishi K, Sparrow JR. Anthocyanins protect against $\mathrm{A} 2 \mathrm{E}$ photooxidation and membrane permeabilization in retinal pigment epithelial cells. Photochem Photobiol 2005; 81: 529-536.

[10] Kalt W, Blumberg JB, McDonald JE, et al. Identification of anthocyanins in the liver, eye and brain of blueberry-fed pigs. J Agric Food Chem 2008; 56: 705-712.

[11] Lee J, Lee HK, Kim CY, et al. Purified high-dose anthocyanoside oligomer administration improves nocturnal vision and clinical symptoms in myopia subjects. Br J Nutr 2005; 93: 895-899.

[12] Matsumoto H, Nakamura Y, Iida H, Ito K, Ohguro H. Comparative assessment of distribution of blackcurrant anthocyanins in rabbit and rat ocular tissues. Exp Eye Res 2006; 83: 348-356.

[13] Abdel-Aal E-S, Abou-Arab AA, Gamel TH, Hucl P, Young JC, Rabalski I. Fractionation of blue wheat anthocyanin compounds and their contribution to antioxidant properties. J Agric Food Chem 2008; 56: 11171-11177.

[14] Astadi IR, Astuti M, Santoso U, Nugraheni PS. In vitro antioxidant activity of anthocyanins of black soybean seed coat in human low density lipoprotein (LDL). Food Chem 2009; 112: 659-663.

[15] Bellido GG, Beta T. Anthocyanin composition and oxygen radical scavenging capacity (ORAC) of milled and pearled purple, black, and common barley. J Agric Food Chem 2009; 57: 1022-1028.

[16] Fukumoto LR, Mazza G. Assessing antioxidant and prooxidant activities of phenolic compounds. J Agric Food Chem 2000; 48: 3597-3604.

[17] Kahkonen MP, Heinonen M. Antioxidant activity of anthocyanins and their aglycones. J Agric Food Chem 2003; 51: 628-633.

[18] Sanchez-Moreno C, Satue-Gracia MT, Frankel EN. Antioxidant activity of selected Spanish wines in corn oil emulsions. J Agric Food Chem 2000; 48: 5581-5587

[19] Colditz GA, Branch LG, Lipnick RJ, et al. Increased green and yellow vegetable intake and lowered cancer deaths in an elderly population. Am J Clin Nutr 1985; 41: 32-36.

[20] Fimognari C, Berti F, Nusse M, Cantelli-Forti G, Hrelia P. Induction of apoptosis in two human leukemia cell lines as well as differentiation in human promyelocytic cells by cyanin-3-O-betaglucopyranoside. Biochem Pharmacol 2004; 67: 2047-2056.

[21] Galvano F, Fauci LL, Vitaglione P, Fogliano V, Vanella L, Felgines C. Bioavailability, antioxidant and biological properties of the natural free-radical scavengers cyanidin and related glycosides. Ann Ist Super Sanita 2007; 43: 382-393.

[22] Hagiwara A, Miyashita K, Nakanishi T, et al. Pronounced inhibition by a natural anthocyanin, purple corn colour, of 2-amino-1methyl-6-phenylimidazo(4,5,-b)pyridine (PhIP)-associated colorectal carcinogenesis in male F344 rats pretreated with 1,2dimethylhydrazine. Cancer Lett 2001; 171: 17-25.

[23] Kang SY, Seeram NP, Nair MG, Bourquin LD. Tart cherry anthocyanins inhibit tumor development in Apc (Min) mice and reduce proliferation of human colon cancer cells. Cancer Lett 2003; 194: 13-19.

[24] Liu Z, Schwimer J, Liu D, Greenway FL, Anthony CT, Woltering EA. Black raspberry extract and fractions contain angiogenesis inhibitors. J Agric Food Chem 2005; 53: 3909-3915.
DeFuria J, Bennett G, Strissel KJ, et al. Dietary blueberry attenuates whole-body insulin resistance in high fat-fed mice by reducing adipocyte death and its inflammatory sequelae. J Nutr 2009; 139: $1-7$.

[26] Guo H, Ling W, Wang Q, et al. Effect of anthocyanin-rich extract from black rice (Oryza sativa L. indica) on hyperlipidemia and insulin resistance in fructose-fed rats. Plant Foods Hum Nutr 2007; 62: 1-6.

[27] Rosenblat M, Hayek T, Aviram M. Anti-oxidative effects of pomegranate juice (PJ) consumption by diabetic patients on serum and on macrophages. Atherosclerosis 2006; 187: 363-371.

[28] Rozenberg O, Howell A, Aviram M. Pomegranate juice sugar fraction reduces macrophage oxidative state, whereas white grape juice sugar fraction increases it. Atherosclerosis 2006; 188: 68-76.

[29] Jayaprakasam B, Vareed SK, Olson LK, Nair MG. Insulin secretion by bioactive anthocyanins and anthocyanidins present in fruits. J Agric Food Chem 2005; 53: 28-31

[30] Matsui T, Ebuchi S, Fukui K, Matsugano K, Terahara N, Matsumoto K. Caffeoylsophorose, a new natural alpha-glucosidase inhibitor, from red vinegar by fermented purple-fleshed sweet potato. Biosci Biotechnol Biochem 2004; 68: 2239-2246.

[31] Zhang Y, Jayaprakasam B, Seeram NP, Olson LK, DeWitt D, Nair MG. Insulin secretion and cyclooxygenase enzyme inhibition by cabernet sauvignon grape skin compounds. J Agric Food Chem 2004; 52: 228-233.

[32] Andriambeloson E, Kleschyov AL, Muller B, Beretz A, Stoclet JC, Andriantsitohaina R. Nitric oxide production and endotheliumdependent vasorelaxation induced by wine polyphenols in rat aorta. Br J Nutr 1997; 120: 1053-1058.

[33] Matheus ME, Fernandes SB, Silveira CS, Rodrigues VP, Menezes FD, Fernandes PD. Inhibitory effects of Euterpe oleracea Mart. on nitric oxide production and iNOS expression. J Ethnopharmacol 2006; 107: 291-296.

[34] Nakamura Y, Matsumoto H, Todoki K. Endothelium-dependent vasorelaxation induced by black currant concentrate in rat thoracic aorta. Jpn J Pharmacol 2002; 89: 29-35.

[35] Stintzing FC, Carle R. Functional properties of anthocyanins and betalins in plants, food, and in human nutrition. Trends Food Sci Technol 2004; 15: 19-38.

[36] Abdel-Aal E-S, Hucl P. A rapid method for quantifiying total anthocyanins in blue aleurone and purple pericarp wheats. Cereal Chem 1999; 76: 350-354.

[37] Castaneda-Ovando A, Pacheco-Hernandez L, Paez-Hernandez E, Rodriguez JA, Galan-Vidal CA. Chemical studies of anthocyanins: A review. Food Chem 2009; 113: 859-871.

[38] Escribano-Bailon MT, Beulga-Santos C, Rivas-Gonzalo JC. Anthocyanins in cereals. J Chromatogr 2004; 1054: 129-141.

[39] Garcia-Viguera C, Zafrilla P, Tomas-Barberan FA. The use of acetone as an extraction solvent for anthocyanins from strawberry fruit. Phytochem Anal 1998; 9: 274-277.

[40] Gil MIG, Tomas-Barberan FA, Hess-Pierce B, Holcroft DM, Kader AA. Antioxidant activity of pomegranate juice and its relationship with phenolic composition and processing. J Agric Food Chem 2000; 48: 4581-4589.

[41] Horbowicz M, Kosson R, Grzesiuk A, Debski H. Anthocyanins of fruits and vegetables - their occurrence, analysis and role in human nutrition. Veg Crops Res Bull 2008; 68: 5-22.

[42] Abdel-Aal E-S, Hucl P. Composition and stability of anthocyanins in blue-grained wheat. J Agric Food Chem 2003; 51: 2174-2180.

[43] Costa CT, Horton D, Margolis SA. Analysis of anthocyanins in foods by liquid chromatography, liquid chromatography-mass spectrometry and capillary electrophoresis. J Chromatogr 2000; 881: 403-410.

[44] Abdel-Aal E-S, Young C, Rabalski I. Anthocyanin composition in black, blue, pink, purple, and red cereal grains. J Agric Food Chem 2006; 54: 4696-4704.

[45] Saenz-Lopez R, Fernandez-Zurbano P, Tena MT. Development and validation of a capillary zone electrophoresis method for the quantitative determination of anthocyanins in wine. J Chromatogr 2003; 990: 247-258.

[46] Calvo D, Saenz-Lopez R, Fernandez-Zurbano P, Tena MT. Migration order of wine anthocyanins in capillary zone electrophoresis Anal Chim Acta 2004; 524: 207-213.

[47] Bednar P, Tomassi AV, Presutti C, Pavlikova M, Lemr K, Fanali S. Separation of structurally related anthocyanins by MEKC. Chromatographia 2003; 58: 283-287. 
[48] Kosir IJ, Kidric J. Use of modern nuclear magnetic resonance spectroscopy in wine analysis: determination of minor compounds. Anal Chim Acta 2002; 458: 77-84.

[49] McGhie TK, Walton MC. The bioavailability and absorption of anthocyanins towards a better understanding. Mol Nutr Food Res 2007; 51: 702-713.

[50] Aura A-M, Martin-Lopez P, O'Leary KA, et al. In vitro metabolism of anthocyanins by human gut microflora. Eur J Nutr 2005; 44: 133-142.

[51] Felgines C, Talavera S, Texier O, Gil-Izquierdo A, Lamaison J-L, Remesy C. Blackberry Anthocyanins Are Mainly Recovered from Urine as Methylated and Glucuronidated Conjugates in Humans. J Agric Food Chem 2005; 53: 7721-7727.

[52] Kay CD, Mazza G, Holub BJ, Wang J. Anthocyanin metabolites in human urine and serum. Br J Nutr 2004; 91: 933-942.

[53] Kay CD, Mazza G, Holub BJ. Anthocyanins exist in the circulation primarily as metabolites in adult men. J Nutr 2005; 135: 25822588.

[54] Keppler K, Humpf H-U. Metabolism of anthocyanins and their phenolic degradation products by the intestinal microflora. Bioorg Med Chem 2005; 13: 5195-5205.

[55] Nielsen ILF, Dragsted LO, Ravn-Haren G, Freese R, Rasmussen SE. Absorption and excretion of black currant anthocyanins in humans and watanabe heritable hyperlipidemic rabbits. J Agric Food Chem 2003; 51: 2813-2820.

[56] Talavera S, Felgines C, Texier O, Besson C, Lamaison J-L, Remesy C. Anthocyanins are efficiently absorbed from the stomach in anesthetized rats. J Nutr 2003; 133: 4178-4182.

[57] Matsumoto H, Inaba H, Kishi M, Tominaga S, Hirayama M, Tsuda T. Orally administered delphinidin-3-rutinoside and cyanidin-3rutinoside are directly absorbed in rats and humans and appear in the blood as the intact forms. J Agric Food Chem 2001; 49: 15461551.

[58] Miyazawa T, Nakagawa K, Kudo M, Muraishi K, Someya K. Direct intestinal absorption of red fruit anthocyanins, cyanidin-3glucoside and cyanidin-3-5-diglucoside, into rats and humans. J Agric Food Chem 1999; 47: 1083-1091.

[59] Joshi SS, Kuszynski CA, Bagchi D. The cellular and molecular basis of health benefits of grape seed proanthocyanidin extract. Curr Pharm Biotechnol 2001; 2: 187-200.

[60] Felgines C, Texier O, Besson C, Fraisse D, Lamaison JL, Rémésy C. Blackberry anthocyanins are slightly bioavailable in rats. J Nutr 2002; 132: 1249-1253.

[61] Cao G, Prior RL. Anthocyanins are detected in human plasma after oral administration of an elderberry extract. Clin Chem 1999; 45: 574-576.

[62] Lapidot T, Harel S, Granit R, Kanner J. Bioavailability of Red Wine Anthocyanins As Detected in Human Urine. J Agric Food Chem 1998; 46: 4297-4302.

[63] Felgines C, Talavéra S, Gonthier M-P, et al. Strawberry Anthocyanins Are Recovered in Urine as Glucuro- and Sulfoconjugates in Humans. J Nutr 2003; 133: 1296-1301.

[64] Tsuda T, Horio F, Osawa T. Absorption and metabolism of cyanidin 3-O- $\beta$-D -glucoside in rats. FEBS Lett 1999; 449: 179-182.

[65] Velioglu YS, Mazza G, Gao L, Oomah BD. Antioxidant activity and total phenolics in selected fruits, vegetables, and grain products. J Agric Food Chem 1998; 46: 4113-4117.

[66] Szabo MR, Iditoiu C, Chambre D, Lupea AX. Improved DPPH determination for antioxidant activity spectrophotometric assay. Chem Papers 2007; 61: 214-216.

[67] Seeram NP, Schutzki R, Chandra A, Nair MG. Characterization, quantification, and bioactivities of anthocyanins in Cornus species. J Agric Food Chem 2002; 50: 2519-2523.

[68] Wang H, Cao G, Prior R. Oxygen radical absorbing capacity of anthocyanins. J Agric Food Chem 1997; 45: 304-309.

[69] Montoro P, Tuberoso CIG, Piacente S, et al. Stability and antioxidant activity of polyphenols in extracts of Myrtus communis L. berries used for the preparation of myrtle liqueur. J Pharmaceut Biomed Anal 2006; 41: 1614-1619.

[70] Muselik J, Garcia-Alonso M, Martin-Lopez MP, Zemlicka M, Rivas-Gonzalo JC. Measurement of antioxidant activity of wine catechins, procyanidins, anthocyanins and pyranoanthocyanins. Int J Mol Sci 2007; 8: 797-809.

[71] Sellappan S, Akoh CC, Krewer G. Phenolic compounds and antioxidant capacity of Georgia-grown blueberries and blackberries. J Agric Food Chem 2002; 50: 2432-2438.
[72] Hopia A, Heinonen M. Antioxidant activity of flavonoids aglycones and their glycosides in methyl linoleate. J Am Oil Chem Soc 1999; 76: 139-144.

[73] Kahkonen MP, Hopia AI, Vuorela HJ, et al. Antioxidant activity of plant extracts containing phenolic compounds. J Agric Food Chem 1999; 47: 3954-3962.

[74] Kahkonen MP, Hopia AI, Heinonen M. Berry phenolics and their antioxidant activity. J Agric Food Chem 2001; 49: 4076-4082.

[75] Lambropoulos I, Roussis IG. Antioxidant activity of red wine phenolic extracts towards oxidation of corn oil. Eur J Lipid Sci Technol 2007; 109: 623-628.

[76] Pike PR, Abdel-Aal E-S M, McElroy AR. Antioxidant activity of oat malt extracts in accelerated corn oil oxidation. J Amer Oil Chem Soc 2007; 84: 663-667.

[77] Cao G, Russell RM, Lischner N, Prior RL. Serum antioxidant capacity is increased by consumption of strawberries, spinach, red wine or vitamin C in elderly women. J Nutr 1998; 128: 2383-2390.

[78] Mazza G, Kay CD, Cottrell T, Holub B. Absorption of anthocyanins from blueberries and serum antioxidant status in human subjects. J Agric Food Chem 2002; 50: 7731-7737.

[79] Youdim KA, Shukitt-Hale B, MacKinnon S, Kalt W, Joseph JA. Polyphenolics enhance red blood cell resistance to oxidative stress: in vitro and in vivo. Biochimica et Biophysica Acta 2000; 1523: 117-122.

[80] Muth ER, Laurent JM, Jasper P. The effect of bilberry nutritional supplementation on night visual acuity and contrast sensitivity. Altern Med Rev 2000; 5: 164-173.

[81] Levy Y, Glovinsky Y. The effect of anthocyanosides on night vision. Eye 1998; 12: 967-969.

[82] Nakaishi H, Matsumoto S, Tominaga M, Hirayama. Effects of blackcurrant anthocyanosides intake on dark adaptation and VDT work-induced transient refractive alternation in healthy humans. Altern Med Rev 2000; 5: 553-562.

[83] Tsuda T, Horio F, Uchida K, Aoki H, Osawa T. Dietary cyanidin 3O-beta-D-glucoside-rich purple corn color prevents obesity and ameliorates hyperglycemia in mice. J Nutr 2003; 133: 2125-2130.

[84] Bonina FP, Leotta C, Scalia G, et al. Evaluation of oxidative stress in diabetic patients after supplementation with a standardised red orange extract. Diab Nutr Metab 2002; 15: 1-19.

[85] Al-Awwadi NA, Araiz C, Bornet A, et al. Extracts enriched in different polyphenolic families normalize increased cardiac NADPH oxidase expression while having differential effects on insulin resistance, hypertension, and cardiac hypertrophy in highfructose-fed rats. J Agric Food Chem 2005; 53: 151-157.

[86] Seibert K, Zhang Y, Leahy K, et al. Pharmacological and biochemical demonstration of the role of cyclooxygenase-2 in inflammation and pain. Proc Natl Acad Sci USA 1994; 95: 13313-13318.

[87] Matsui T, Ueda T, Oki T, Sugita K, Terahara N, Matsumoto K. Alpha-glucosidase inhibitory action of natural acylated anthocyanins. 1. Survey of natural pigments with potent inhibitory activity. J Agric Food Chem 2001; 49: 1948-1951.

[88] Fitzpatrick DF, Hirschfield SL, Coffey RG. Endotheliumdependent vasorelaxing activity of wine and other grape products. Am J Physiol 1993; 265: H774-H778.

[89] Serraino I, Dugo L, Dugo P, et al. Protective effects of cyanidin-3$\mathrm{O}$-glucoside from blackberry extract against peroxynitriteinduced endothelial dysfunction and vascular failure. Life Sci 2003; 73: 1097-1114.

[90] Wallerath T, Li H, Godtel-Ambrust U, Schwarz PM, Forstermann U. A blend of polyphenolic compounds explains the stimulatory effect of red wine on human endothelial NO synthase. Nitric Oxide 2005; 12: 97-104.

[91] Liu M, Li XQ, Weber C, et al. Antioxidant and antiproliferative activities of raspberries. J Agric Food Chem 2002; 50: 2926-2930.

[92] Serafino A, Sinibaldi-Vallebona P, Lazzarino G, et al. Differentiation of human melanoma cells induced by cyanidin-3-O-betaglucopyranoside. Faseb J 2004; 18: 1940-1942.

[93] Magnuson BA, Lala G, Kwon YJ, et al. Anthocyanin-rich extracts inhibit growth of human colon cancer cells and azoxymethaneinduced colon aberrant crypt in rats: implications for colon cancer chemoprevention. Cancer Epidemiol Biomark Prev 2003; 12 : 1323S-1324S.

[94] Harris GK, Gupta A, Nines RG, et al. Effects of lyophilized black raspberries on azoxymethane-induced colon cancer and 8-hydroxy2'-deoxyguanosine levels in the Fischer 344 rat. Nutr Cancer 2001; 40: $125-133$. 
[95] Afaq F, Saleem M, Krueger CG, Reed JD, Mukhtar H. Anthocyanin- and hydrolyzable tannin-rich pomegranate fruit extract modulates MAPK and NF-kappaB pathways and inhibits skin tumorigenesis in CD-1 mice. Int J Cancer 2005; 113: 423-433.

[96] Adlercreutz H. Epidemiology of phytoestrogens. Baillieres Clin Endocrinol Metab 1998; 12: 605-623.

[97] Almendingen K, Hofstad B, Vatn MH. Dietary habits and growth and recurrence of colorectal adenomas: results from a three-year endoscopic follow-up study. Nutr Cancer 2004; 49: 131-138.

[98] Klatsky AL. Epidemiology of coronary heart disease--influence of alcohol. Alcohol Clin Exp Res 1994; 18: 88-96.

[99] Visioli F, Galli C. The role of antioxidants in the Mediterranean diet. Lipids 2001; 36: S49-S52.

[100] Wollin SD, Jones PJH. Alcohol, red wine and cardiovascular disease. J Nutr 2001; 131: 1401-1404.

[101] Matsumoto M, Hara H, Chiji H, Kasai T. Gastroprotective effect of red pigments in black chokeberry fruit (Aronia melanocarpa Elliot) on acute gastric hemorrhagic lesions in rats. J Agric Food Chem 2004; 52: 2226-2229.

[102] Morazzoni P, Magistretti MJ. Effects of Vaccinium myrtillus anthocyanosides on postacyclin-like activity in rat arterial tissue. Fitoterapia 1986; 57 : 11-14

[103] Akhmadieva AK, Zaichkina SI, Ruzieva RK, Ganassi EE. The protective action of a natural preparation of anthocyanin (pelargonidin-3,5-diglucoside). Radiobiologiia 1993; 33: 433-435.

[104] Yoshinaga K. Liquor with pigments of red rice. J Brew Soc Jpn 1986; 81: 337-342.

[105] Stintzing FC, Stintzing AS, Carle R, Frei B, Wrolstad RE. Color and antioxidant properties of cyanidin-based anthocyanin pigments. J Agric Food Chem 2002; 50: 6172-6180.
[106] Boulton R. The copigmentation of anthocyanins and its role in the color of red wine: a critical review. Am J Enol Vitic 2001; 52: 6787.

[107] Hofmann T. Studies on the influence of the solvent on the contribution of single maillard reaction products to the total color of browned pentose/alanine solutions - A quantitative correlation using the color activity concept. J Agric Food Chem 1998; 46: 39123917.

[108] Ma J, Li Y, Li J, et al. Constituents of red yeast rice, a traditional Chinese food and medicine. J Agric Food Chem 2000; 48: 52205225.

[109] Bridle P, Timberlake CF. Anthocyanins as natural food colours selected aspects. Food Chem 1996; 58: 103-109.

[110] Wrolstad RE. Anthocyanin pigments - bioactivity and coloring properties. J Food Sci 2004; 69: C419-C425.

[111] Bezar HJ. Konini, specialty bread wheat. N.Z. Wheat Rev 1982; 15: 62-63.

[112] Awika JM, Rooney LW, Waniska RD. Anthocyanins from black sorghum and their antioxidant properties. Food Chem 2004; 90: 293-301.

[113] Amazon Botanicals [homepage on the internet]. Newark, DE: Amazon Botanicals, LLC.; c2005-2009 [cited 2009 Oct 15]. Available from: http://www.amazon-botanicals.com/Anthocyanins_s/ 63.htm

[114] Biolink Group [homepage on the internet]. Sandnes, NO: Biolink Group, AS.; [cited 2009 Oct 15] Available from: http://www. biolink.no/products/professional-dietary-supplement-article86111.html

[115] Pharmanex [homepage on the internet]. Provo, UT: Pharmanex, Inc.; [cited 2009 Oct 15]. Available from: http://www.pharmanex. com/corp/product/solutions/cholestin.shtml

(C) Shipp and Abdel-Aal; Licensee Bentham Open .

This is an open access article licensed under the terms of the Creative Commons Attribution Non-Commercial License (http://creativecommons.org/licenses/by-nc/3.0/) which permits unrestricted, non-commercial use, distribution and reproduction in any medium, provided the work is properly cited. 